\title{
Development of a Fatigue Damage Propagation Model for Fibre Metal Laminates with Locally Tailored Fibre Layers
}

by

Jeremy Atkinson

A Thesis submitted to

the Faculty of Graduate Studies and Research

in partial fulfilment of

the requirements for the degree of

Master of Applied Science

Aerospace Engineering

Ottawa-Carleton Institute of Mechanical

and Aerospace Engineering

Carleton University

Ottawa, Ontario, Canada

July 2014

Copyright (C)

2014 - Jeremy Atkinson 


\section{Abstract}

Previous models of fatigue crack growth in fibre metal laminates (FMLs) have proven successful in characterizing the behaviour of generic FMLs, however current models are limited to uniform, symmetric laminates. The goal of this project was to develop, implement, validate, and verify a simple analytical model for crack propagation within an FML with non-uniform stiffness, with principal focus on GLARE. The motivation for this research lies in expanding existing research on local substitution of glass/epoxy fibre with stiffer carbon/epoxy material. It was found that this local stiffness modification can act as a crack arresting feature - as such, characterization of the exact behaviour would be quite useful in future applications.

The model presented in this thesis was implemented in the MATLAB ${ }^{\mathrm{TM}}$ environment

and is based on previous analytical work by Alderliesten. Experimental validation and verification of the model showed good correlation between measured and predicted strains in the fibre and metal layers, good correlation between measured and predicted fatigue crack growth rates, and accurate prediction of the delamination shape close to the crack tip. 


\section{Acknowledgments}

We stand on the shoulders of giants. I would like to thank the many technicians and researchers involved in fibre metal laminate development for having laid the groundwork for this investigation. In particular, I would like to thank:

- My supervisors, Calvin Rans - for believing in me and supporting me through a stressful thesis, and Craig Merrett, for taking me on and for his indispensable advice regarding thesis writing.

- Cees Paalvast, Bob de Vogel, Berthil Grashof and the whole team of technicians at TUDelft's DASML. Your aid in guiding a young researcher through the testing process was very much appreciated.

- The Department of Aerospace Structures and Materials at TUDelft for the generous donation of shop and equipment time, materials, and expertise.

- Suzanne, for always being a good friend when I needed it most.

- My friends and colleagues Josh, Alex, Anne, Sherif, and Owen. You kept me sane during the most stressful times.

- My partner Rebecca for always supporting me and tolerating my frequent late nights spent debugging code. 


\section{Table of Contents}

Abstract

Acknowledgments

Table of Contents $\quad$ iv

List of Tables $\quad$ viii

$\begin{array}{ll}\text { List of Figures } & \text { ix }\end{array}$

Nomenclature $\quad$ xii

1 Introduction 1

2 Crack Growth in Fibre Metal Laminates 3

2.1 Fibre metal laminates. . . . . . . . . . . . . . . . . 4

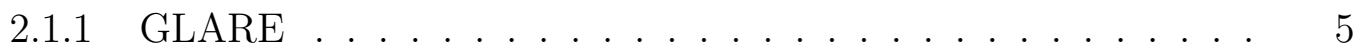

2.1.2 Fibre Metal Laminate Fatigue Behaviour . . . . . . . . 6

2.2 Fibre metal laminate fatigue crack prediction methodologies $\ldots . .8$ 
2.2.1 Empirical models . . . . . . . . . . . . . . . . . . . 8

2.2.2 Mechanistic models . . . . . . . . . . . . . . . . . . . 9

2.3 Novel GLARE concepts . . . . . . . . . . . . . . . . . . . . . . . . 11

3 Project Definition $\quad 13$

3.1 Project Scope . . . . . . . . . . . . . . . . . . 14

3.1.1 Modelling ..................... 14

3.1 .2 Materials ... . . . . . . . . . . . . . . . 14

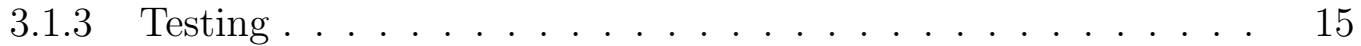

4 Implementation of the Modified Damage Growth Prediction Model 16

4.1 Introduction . . . . . . . . . . . . . . . . . 16

4.2 Numerical Implementation of the Alderliesten Crack Growth Model . 18

4.2.1 Paris Relations for Damage Growth Prediction . . . . . . . . . 20

4.2.2 Superposition of Far Field and Bridging . . . . . . . . . . . 21

4.2.3 Displacement Compatibility . . . . . . . . . . . . . . 22

4.3 Implementation of Variation of Fibre Stiffness Ahead of Crack Tip . . 27

4.4 Implementation of Variation of Fibre Stiffness Behind Crack Tip . . . 28

4.5 Overall Model Implementation . . . . . . . . . . . . . . . . . 29

5 Model Validation and Verification $\quad 36$

5.1 Introduction . . . . . . . . . . . . . . . . 36 
5.2 Testing and Simulation Parameters . . . . . . . . . . . . . 37

5.3 Comparison of updated model to original Alderliesten model . . . . . 37

5.3.1 Crack opening displacement validation . . . . . . . . . . 38

5.3.2 Fatigue crack growth model validation . . . . . . . . . . . 39

5.3.3 Delamination shape validation . . . . . . . . . . . . . . . 42

5.4 Validation of load redistribution model . . . . . . . . . . . . . . 43

5.5 Verification .......................... 47

5.5.1 Crack growth rate verification . . . . . . . . . . . 47

5.5.2 Delamination Shape Verification . . . . . . . . . . . . 51

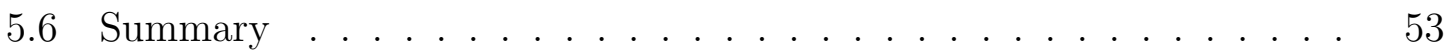

6 Conclusions and Future Work $\quad 56$

6.1 Conclusions ......................... 56

6.2 Future Work . . . . . . . . . . . . . . . . 57

List of References $\quad 59$

$\begin{array}{ll}\text { Appendix A Material Properties } & 61\end{array}$

$\begin{array}{lll}\text { Appendix B } & \text { Test Specimens } & 62\end{array}$

Appendix C Crack Growth Data $\quad 64$

Appendix D Crack Growth Constants from Schivje $\quad 71$ 
Appendix E Carbon Fibre Delamination Constant Data 


\section{List of Tables}

2.1 GLARE grades and properties (reproduced from [2]) . . . . . . . 5

5.1 Model Inputs and Testing Parameters . . . . . . . . . . . . . . 37

A.1 Properties of GLARE and stiffener constituent materials . . . . . . 61

C.1 ASTM Crack Growth Data for Carbon-Glass-Carbon Panel, $W=$ $85 / 80 / 85, \sigma_{\max }=140 M P a, R=0.10,2 a_{0}=15 m m \ldots 64$

C.2 ASTM Crack Growth Data for Glass-Carbon-Glass Panel, $W=$ $85 / 80 / 85, \sigma_{\max }=140 M P a, R=0.10,2 a_{0}=15 \mathrm{~mm} \ldots \ldots 6$

D.1 Crack growth data from Schijve $[17] \ldots \ldots$. . . . . . . . . 72

E.1 Delamination growth test data for carbon fibre . . . . . . . . . . 74 


\section{List of Figures}

1.1 Generic fibre metal laminate layup $[1] \ldots \ldots \ldots \ldots$

2.1 Damage tolerance, initial damage, and critical damage state definitions 3

2.2 Crack growth curve of aluminium 2024-T3 and GLARE 3-3/3-0.3L and GLARE 4B-4/3-0.5LT for constant amplitude fatigue loading [6] . . 6

2.3 Fibre bridging in fibre metal laminates (adapted from [7]) . . . . . 7

2.4 Internal tear strap in GLARE $[16] \ldots \ldots \ldots \ldots \ldots \ldots$

2.5 Effect of an internal tear strap on fatigue crack growth in GLARE [16] 12

4.1 Generalized Crack and Delamination Geometry . . . . . . . . . . 19

4.2 Fibre Bridging (Adapted from $[7]) \ldots \ldots \ldots$

4.3 Displacement Compatibility (Modified from $[16]$ ) $\ldots \ldots \ldots$

4.4 Model Implementation - Simulation Flow . . . . . . . . . . . . 30

5.1 Comparison between predicted (original Alderliesten model and updated model) and measured crack opening of GLARE 3-6/5-0.4 with $W=100 \mathrm{~mm}, \sigma_{\max }=100 \mathrm{MPa}, R=0.05,2 a_{0}=3 \mathrm{~mm}$. Constructed with test data from $[7] \ldots \ldots \ldots \ldots \ldots \ldots$ 
5.2 Comparison between predicted (original Alderliesten model and updated model) and crack growth rates for GLARE 3-4/3-0.5 with $W=500 \mathrm{~mm}, \sigma_{\max }=100 \mathrm{MPa}, R=0.05,2 a_{0}=75 \mathrm{~mm}$. Constructed

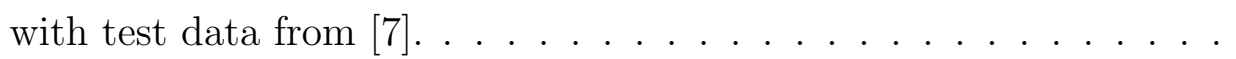

5.3 Comparison between predicted (original Alderliesten model and updated model) and crack growth rates for GLARE 3-4/3-0.5 with $W=500 \mathrm{~mm}, \sigma_{\max }=120 \mathrm{MPa}, R=0.05,2 a_{0}=75 \mathrm{~mm}$. Constructed with test data from $[7] \ldots \ldots \ldots \ldots \ldots \ldots$

5.4 Comparison between predicted (original Alderliesten model and updated model) and crack growth rates for GLARE 3-6/5-0.4 with $W=100 \mathrm{~mm}, \sigma_{\max }=100 \mathrm{MPa}, R=0.05,2 a_{0}=3 \mathrm{~mm}$. Constructed with test data from $[7] \ldots \ldots \ldots \ldots \ldots \ldots$

5.5 Comparison between measured and predicted (Alderliesten and updated model) delamination sizes for GLARE 3-4/3-0.5 with $W=$ $500 \mathrm{~mm}, \sigma_{\max }=120 \mathrm{MPa}, R=0.05,2 a_{0}=75 \mathrm{~mm}$. Constructed with test data from [7] . . . . . . . . . . . . . . . . .

5.6 Comparison of predicted and measured fibre layer strains for CarbonGlass-Carbon configuration ITS-GLARE 2A-2/1-0.3 with $W=$ $85 / 80 / 85 m m, \sigma_{\max }=140 M P a, R=0.1,2 a_{0}=15 m m, N=300000$.

5.7 Comparison of predicted and measured fibre layer strains for GlassCarbon-Glass configuration ITS-GLARE 2A-2/1-0.3 with $W=$ $85 / 80 / 85 \mathrm{~mm}, \sigma_{\max }=140 \mathrm{MPa}, R=0.1,2 a_{0}=15 \mathrm{~mm}, N=1000000$.

5.8 Comparison of predicted and measured fibre layer strains for CarbonGlass-Carbon configuration ITS-GLARE 2A-2/1-0.3 with $W=$ $85 / 80 / 85 \mathrm{~mm}, \sigma_{\max }=140 M P a, R=0.1,2 a_{0}=15 \mathrm{~mm}, N=1000000$. 
5.9 Comparison of predicted and measured crack growth rates for CarbonGlass-Carbon configuration ITS-GLARE 2A-2/1-0.3 with $W=$ $85 / 80 / 85 \mathrm{~mm}, \sigma_{\max }=140 M P a, R=0.1,2 a_{0}=15 \mathrm{~mm} . \ldots \ldots 48$

5.10 Comparison of predicted and measured crack growth rates for Glass-Carbon-Glass configuration ITS-GLARE 2A-2/1-0.3 with $W=$ $85 / 80 / 85 \mathrm{~mm}, \sigma_{\max }=140 M P a, R=0.1,2 a_{0}=15 \mathrm{~mm} . \ldots \ldots$

5.11 Crack growth rate comparison from literature [16] for Glass-CarbonGlass-Carbon-Glass configuration ITS-GLARE 3-3/2-0.4 with $W=$ $50 / 25 / 100 / 25 / 50 \mathrm{~mm}, \sigma_{\max }=120 M P a, R=0.05,2 a_{0}=25 \mathrm{~mm} . \ldots \quad 50$

5.12 Comparison of predicted and measured delamination sizes for CarbonGlass-Carbon configuration ITS-GLARE 2A-2/1-0.3 with $W=$ $85 / 80 / 85 \mathrm{~mm}, \sigma_{\max }=140 \mathrm{MPa}, R=0.1,2 a_{0}=15 \mathrm{~mm}, 2 a_{f}=104 \mathrm{~mm}$,

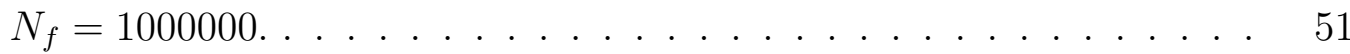

5.13 Comparison of predicted and measured delamination sizes for GlassCarbon-Glass configuration ITS-GLARE 2A-2/1-0.3 with $W=$ $85 / 80 / 85 \mathrm{~mm}, \sigma_{\max }=140 \mathrm{MPa}, R=0.1,2 a_{0}=15 \mathrm{~mm}, 2 a_{f}=112 \mathrm{~mm}$,

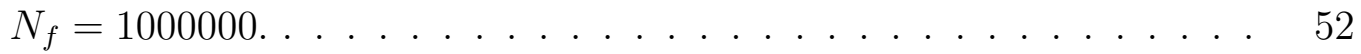

B.1 Carbon-Glass-Carbon Fatigue Test Specimen Configuration . . . . . . 62

B.2 Glass-Carbon-Glass Fatigue Test Specimen Configuration . . . . . . .

D.1 Crack growth constants for Al 2024-T3 calculated using data from Schivje $[17] \ldots \ldots \ldots \ldots \ldots \ldots \ldots$

E.1 Delamination growth constants for carbon fibre . . . . . . . . 73 


\section{Nomenclature}

\begin{tabular}{|c|c|c|}
\hline Symbol & Definition & [units] \\
\hline$K$ & Stress intensity factor & {$[M P a \sqrt{m m}]$} \\
\hline$s$ & Sawcut length & {$[\mathrm{mm}]$} \\
\hline$a$ & Crack length & {$[m m]$} \\
\hline$b$ & Delamination length & {$[m m]$} \\
\hline$C$ & Empirical Paris constant & \\
\hline$m$ & Empirical Paris exponent & \\
\hline$R$ & Stress ratio & {$[M P a / M P a]$} \\
\hline$G_{I I}$ & Mode II Strain energy release rate & {$\left[M P a^{2} m m\right]$} \\
\hline$N$ & Number of cycles & \\
\hline$n$ & Number of layers & \\
\hline$t$ & Layer thickness & {$[m m]$} \\
\hline$W$ & Specimen width & {$[m m]$} \\
\hline$E$ & Young's Modulus & {$[G P a]$} \\
\hline
\end{tabular}




\section{Symbol Definition}

[units]

$j \quad$ Number of shear interfaces

$\sigma \quad$ Stress

$[M P a]$

$w \quad$ Delamination zone width

$[\mathrm{mm}]$

$\nu \quad$ Poisson ratio

$v \quad$ Crack opening displacement

$[m m]$

$\delta \quad$ Deformation

$[m m]$

$\tau \quad$ Shear stress

$[M P a]$

G Shear Modulus

$[G P a]$

$P \quad$ Load

$[N]$

$L \quad$ Specimen length

$[L]$ 


\section{Chapter 1}

\section{Introduction}

"The art of moulding materials we do not really understand into shapes we cannot really analyze, so as to withstand forces we cannot really assess, in such a way that the public does not really suspect." Dr. E. H. Brown, speaking on structural engineering.

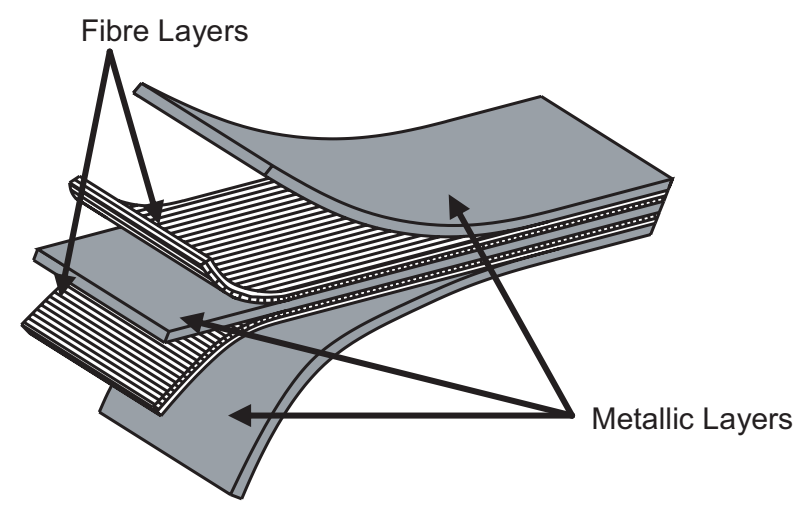

Figure 1.1: Generic fibre metal laminate layup [1]

Over the past four decades, research has been carried out at the Delft University of Technology on fibre metal laminates (an example of which is seen in Figure 1.1), culminating in the development of GLARE (Glass Laminate Aluminium Reinforced Epoxy) in the late 1980s [2]. GLARE has seen marked success as a highly damage 
tolerant aerospace material, with major applications including Airbus' A380 upper fuselage and stabiliser leading edges, the Learjet 45's forward bulkhead, and cargo doors in certain Boeing 777's [3].

Recently, research in fibre metal laminates has been trending away from standard GLARE grades and towards tailored structures. With this tailoring comes the challenge of accurately predicting fatigue crack propagation within a varying structure. Work has been completed towards expanding preexisting models to account for unsymmetric laminates [4]. As well, preliminary investigations as to the effectiveness of a localized increase in fibre stiffness have shown promise in implementation as a crack arresting feature.

This thesis' investigation aims to address a gap in fatigue crack growth propagation prediction techniques: that of variations in fibre stiffness along the width of a panel. The objective of this investigation is to model the effect of the following cases on fatigue crack growth in fibre metal laminates:

1. A variation in stiffness in the fibre layers ahead of the crack tip

2. A variation in stiffness in the fibre layers behind the crack tip

Chapter 2 presents a brief background on the fatigue behaviour of fibre metal laminates and current prediction methodologies. Following this, Chapter 3 lays out the project definition and scope. Chapter 4 then presents the baseline Alderliesten fatigue crack growth model for fibre metal laminates and details the modifications required to accommodate variations in stiffness in the fibre layers of the laminate. Chapter 5 contains the experimental validation and verification of the implemented model. The final chapter presents the conclusions and proposed future work. 


\section{Chapter 2}

\section{Crack Growth in Fibre Metal}

\section{Laminates}

The damage tolerant design philosophy is heavily used in aerospace structural design [5]. Damage tolerance is founded on the principle that from construction, flaws and damage are present within a structure. As the structure progresses through its service life, this damage starts to propagate and eventually the structure cannot sustain its maximum design loads. This damage state is referred to as a critical damage state and is illustrated in Figure 2.1.

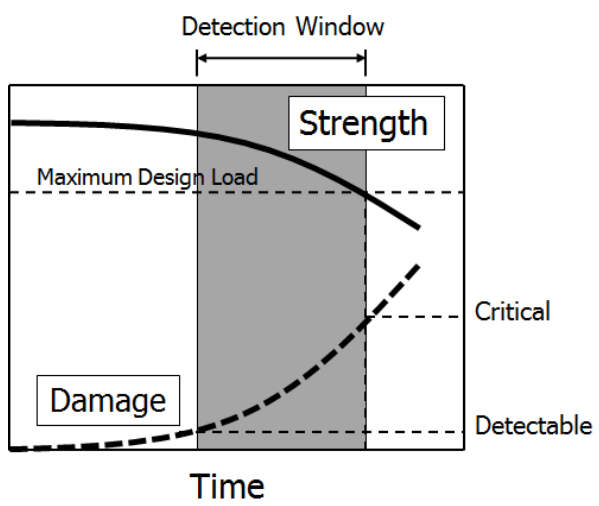

Figure 2.1: Damage tolerance, initial damage, and critical damage state definitions 
The interval of time from the detection of the damage through to the damage reaching its critical state is referred to as a detection window. This window is then used to determine the inspection and maintenance intervals for structures. Understanding of damage propagation and residual strength of the materials used in the structure allows the designer to optimize the structure for the maximum design loads. A complete comprehension of damage propagation and loading effects gives designers the ability to accurately specify intervals for inspection and maintenance.

The aim of this thesis is to extend the crack growth prediction capabilities for Fibre Metal Laminates (FMLs). However, first a brief introduction into FMLs and the state of the art in crack growth prediction for these materials is required.

\subsection{Fibre metal laminates}

Fibre metal laminates are a highly damage tolerant composite material developed at the Delft University of Technology. They find their origins in early work on laminated metallic structures studied by Fokker in the late 1940s [2]. Initial investigations into the fatigue performance of tailored laminated aluminium structures found that these structures had excellent fatigue performance over their monolithic counterparts. This was due to the cracks initiating in individual layers, with the intact fibre layers bridging the crack and slowing the damage propagation.

Eventually, several researchers [2] thought of integrating fibres into the adhesive layers

- first nylon fibres, then experiments with carbon fibres were carried out. Finally, the use of aramid fibres led to the creation of ARALL ${ }^{\mathrm{TM}}$, which achieved limited application in the aerospace field [2]. The culmination of this research into fibre metal laminates to date has been the invention of GLARE. 


\subsubsection{GLARE}

GLARE (Glass Laminate Aluminium Reinforced Epoxy) is a class of fibre metal laminates developed at the Delft University of Technology in the 1980s [2]. This particular fibre metal laminate consists of aluminium sheets laminated with continuous glass fibre layers, creating a lightweight, damage tolerant structure. GLARE is divided into different categories (grades) depending on the ratio of fibre-to-metal layers and the fibre layer orientations (see Table 2.1 for a list). Each GLARE grade has certain strengths to which it has been tailored (for example, GLARE 3 is especially good in impact and fatigue).

Table 2.1: GLARE grades and properties (reproduced from [2])

\begin{tabular}{|c|c|c|c|c|}
\hline GLARE & $\begin{array}{l}\text { Sub- } \\
\text { grade }\end{array}$ & $\begin{array}{l}\text { Metal sheet thick- } \\
\text { ness }[\mathrm{mm}] \& \text { alloy }\end{array}$ & $\begin{array}{l}\text { Prepreg orientation in } \\
\text { each fibre layer }\end{array}$ & $\begin{array}{l}\text { Main beneficial } \\
\text { characteristics }\end{array}$ \\
\hline GLARE1 & & 0.3-0.4 7475-Т671 & $0 / 0$ & $\begin{array}{l}\text { fatigue, strength, } \\
\text { yield stress }\end{array}$ \\
\hline GLARE2 & $\mathrm{A}$ & 0.2-0.5 2024-T3 & $0 / 0$ & fatigue, strength \\
\hline & $\mathrm{B}$ & 0.2-0.5 2024-T3 & $90 / 90$ & fatigue, strength \\
\hline GLARE3 & & 0.2-0.5 2024-Т3 & $0 / 90$ & fatigue, impact \\
\hline GLARE4 & $\mathrm{A}$ & 0.2-0.5 2024-T3 & $0 / 90 / 0$ & $\begin{array}{l}\text { fatigue, strength in } \\
0^{\circ} \text { direction }\end{array}$ \\
\hline & $\mathrm{B}$ & 0.2-0.5 2024-T3 & $90 / 0 / 90$ & $\begin{array}{l}\text { fatigue, strength in } \\
90^{\circ} \text { direction }\end{array}$ \\
\hline GLARE5 & - & 0.2-0.5 2024-T3 & $0 / 90 / 90 / 0$ & impact \\
\hline GLARE6 & $\mathrm{A}$ & 0.2-0.5 2024-T3 & $+45 /-45$ & $\begin{array}{l}\text { shear, off-axis } \\
\text { properties }\end{array}$ \\
\hline & $\mathrm{B}$ & $0.2-0.52024-\mathrm{T} 3$ & $-45 /+45$ & $\begin{array}{l}\text { shear, off-axis } \\
\text { properties }\end{array}$ \\
\hline
\end{tabular}

The development of GLARE grades arose out of certification constraints surrounding the application of the material technology to aircraft structures. By developing fixed grades with standardized properties, FMLs could be treated as a metallic material 
during certification due to several commonalities with metal, including plasticity and corrosion behaviour [2]. Although this decision made the certification of the material easier, it had the unfortunate consequence of limiting exploitation of the tailorable composite nature of the material.

\subsubsection{Fibre Metal Laminate Fatigue Behaviour}

Continuous fibre metal laminates (such as GLARE) exhibit extremely good fatigue crack growth behaviour. While monolithic metals such as aluminium experience exponential crack growth rates with increasing crack length (as seen with general Paris relations), crack growth rates in GLARE vary almost linearly with crack length (Figure 2.2). This is due to the mechanisms driving the crack and the behaviour of the fibre layers.

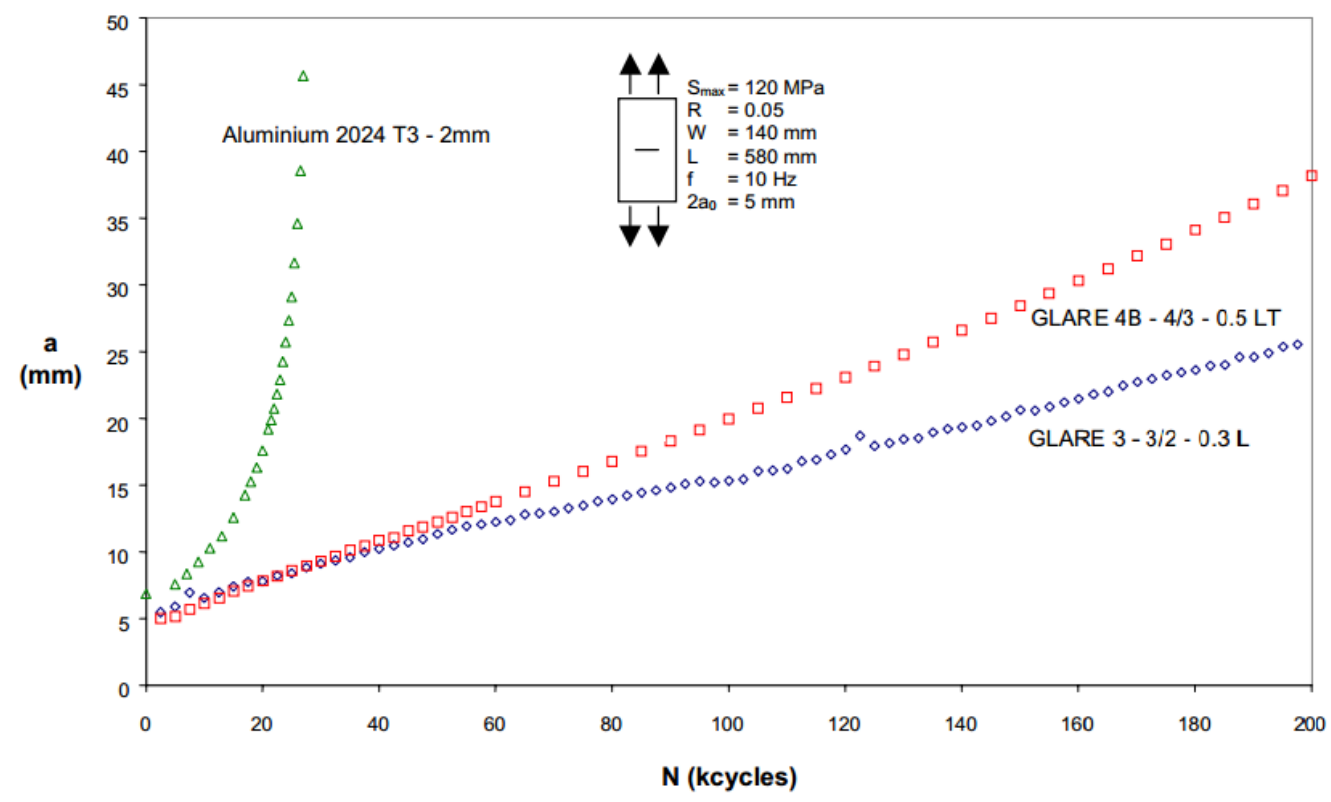

Figure 2.2: Crack growth curve of aluminium 2024-T3 and GLARE 3-3/3-0.3L and GLARE 4B-4/3-0.5LT for constant amplitude fatigue loading [6]

As a crack grows through an FML, the fibre layers remain intact and bridge the 
crack. The matrix material of the fibre layers also begins to fail perpendicular to the crack front, causing a delamination in the fibre layers. The intact fibres along these delamination zones bridge the crack, constraining its opening and reducing the load at the crack tip (Figure 2.3). This fibre bridging results in a decrease in crack growth rates and an increase in the specimen life. The increased bridging load in the fibre layer increases the rate at which the delamination propagates through the fibre layers. This results in a coupled damage - both the delaminations in the fibre layers and the cracks in the metal layers influence each other, resulting in a very damage tolerant material.

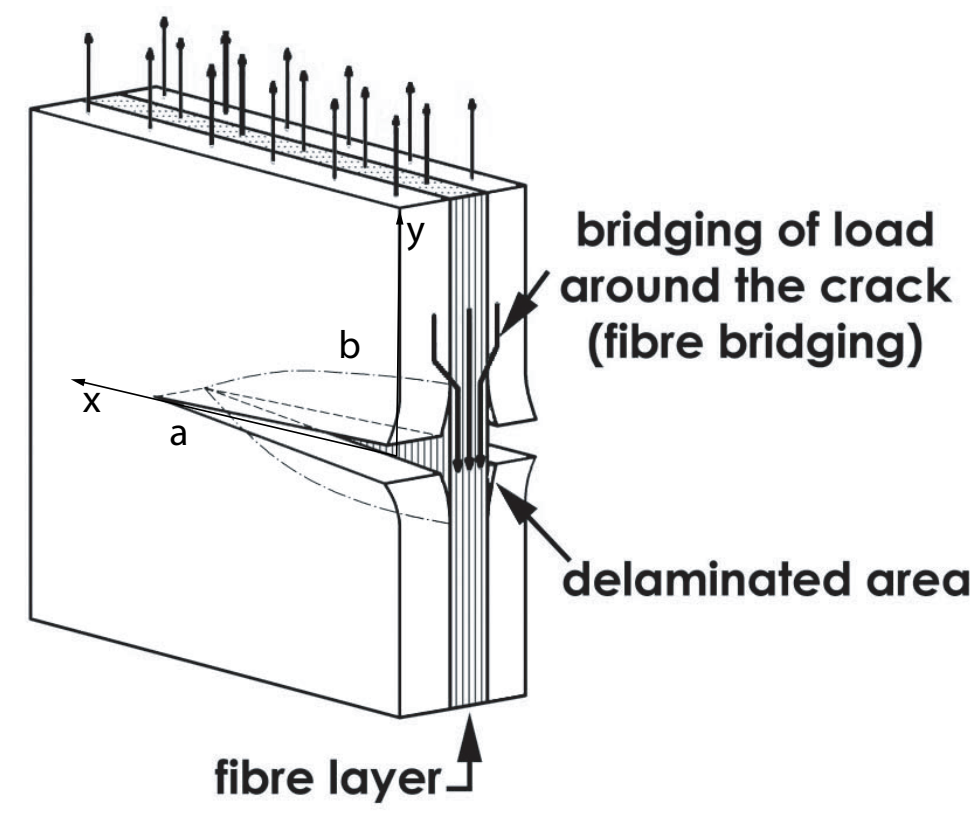

Figure 2.3: Fibre bridging in fibre metal laminates (adapted from [7])

Further information on fibre metal laminates (in particular GLARE) can be found in a wide array of sources, however Ad Vlot's "Fibre metal laminates: an introduction" [2] does an excellent job of surveying the strengths and major features of these structures. 


\subsection{Fibre metal laminate fatigue crack prediction methodologies}

There exist many damage prediction models for fatigue crack growth in fibre metal laminates. The majority of models can be divided into two major categories: empirical and mechanistic models [8]. These two categories share one characteristic they employ empirical Paris relations to calculate the fatigue crack growth rates. In essence, the differentiation between models exists in the methodologies used to calculate the stress intensity factors.

\subsubsection{Empirical models}

Empirical models, seek to characterize fatigue crack growth by observations, determining stress intensity factors phenomenologically. These stress intensity factors are used to calculate the fatigue crack growth rate by way of Paris relations. Reviewed for this investigation were the methods of Takamatsu et al. [9], Guo \& Wu [10], and Cox [11]. In these cases, the researchers approached the problem from an engineering perspective, treating the laminated structure as a monolithic material with behaviour defined in terms of the constituent metal.

Most empirical fatigue crack growth models for fibre metal laminates take the form seen in Equation 2.1, where $\beta_{f m l}$ is an empirical correction factor for the laminate configuration being studied, $a$ is the current crack length, and $\sigma_{\text {applied }}$ is the load applied to the specimen. $\Delta K_{f m l}$ is the equivalent change in stress intensity factor for the fibre metal laminate, which is used to calculate the crack growth rate using an empirical Paris relation. 


$$
\Delta K_{f m l}=\beta_{f m l} \Delta \sigma_{\text {applied }} \sqrt{\pi a}
$$

Generally, these models derive the empirical correction factors by treating the laminated structure as a monolithic material, combining the material constants for the constituent materials and the specimen's geometric configuration.

The empirical correction factors derived for these models differ greatly in format and derivation; however, they all share the same weakness: reliance on previously performed tests for the specific specimen configuration. As such, the models developed using these methodologies often lack robustness, particularly when attempting to accommodate non-uniform laminates.

A further limitation of these empirical models is that fatigue crack growth rates within fibre metal laminates are dependent both on specimen geometry as well as on delamination shape [7]. As initial damage states can vary, this further reduces the accuracy of empirical models. Furthermore, the majority of these models were validated with limited experimental testing (Guo \& Wu's model [10] had a mere 15 test specimens).

While the final models may produce accurate results for the laminate being studied, the end conclusion is that these models lack robustness and tailorability to new materials/configurations.

\subsubsection{Mechanistic models}

"All other things being equal, mechanistic models are more powerful since they tell you about the underlying processes driving patterns. They are more likely to work correctly when extrapolating beyond the observed 
conditions." [12]

Mechanistic models seek to characterize the stress intensity factor at the crack tip using fracture mechanics, specimen geometry, and the current crack and delamination geometry. This stress intensity factor is then used to calculate the crack growth rate by way of an empirical Paris relation. Many of these models make use of numerical implementations of the analytical model for practical purposes.

The principal mechanistic models investigated in this work are those of Marissen [13], Guo \& Wu [14], Alderliesten [7], and Wilson [4]. Here, as opposed to the engineering approach taken with empirical models, mechanistic models seek to explain the underlying processes driving the crack growth. These models include the effects of delamination shape and size, the specimen geometry, and the load configuration.

The method of Marissen is among the oldest model, having been developed in the late 1980s [13]. It was derived for use with ARALL ${ }^{\mathrm{TM}}$. This model uses a superposition of stress intensity factors, derived from the far field applied loads in the aluminium layer and the adhesive shear deformation. This superposition is then used to derive crack growth rates using an empirical Paris relation. Marissen's model had one major limitation, that it assumed an elliptical delamination shape, which was shown to be incorrect by Alderliesten [15]. Despite this limitation, Marissen's model presents a good approximation of the crack growth mechanisms in FMLs.

Guo \& Wu's methodology is similar to that of Marissen, in that they made use of a superposition of stress intensity factors to calculate the fatigue crack growth rate. Their superposition consisted of a far-field stress intensity factor derived for the metal layers and a bridging stress intensity factor determined by a displacement compatibility condition across the crack. The aluminium crack opening, consisting of the crack opening under applied load and the bridging stress, was equated to the physical crack opening displacement, consisting of the fibre elongation under the applied load, 
the adhesive layer shear deformation and the aluminium deformation. Unfortunately, Guo \& Wu's methodology also assumed a delamination shape, studying the effect of both triangular and elliptical delamination shapes [14]. The true delamination shape in fibre metal laminates in fact was found to be a shape in between triangular and elliptical [7].

In general, the primary advantage of mechanistic models is that they minimize the required inputs. As a result, mechanistic models are significantly more robust, and with proper testing, much more flexible in terms of material, geometry, and load configurations. Of the mechanistic models researched, Alderliesten's model was found to be the most robust and was selected as a baseline model for this investigation. The model is explained in detail in Chapter 4.

\subsection{Novel GLARE concepts}

To date, GLARE and other fibre metal laminates have seen extensive use in the aerospace industry as a primary structural material [2]. In recent years, focus has shifted to tailorability. Proper characterization of fatigue behaviour is essential for proper optimization of FML structures for aerospace applications. To this effect, work has focused on fatigue behaviour of non-symmetric and variable stiffness fibre metal laminates. Wilson [4] built upon Alderliesten's work in creating a more robust fatigue crack growth model able to predict crack propagation through unsymmetric laminates.

An initial investigation [16] into variable stiffness laminates showed promise for tailoring of fibre layers, particularly as internal tear straps for crack arrest (Figure 2.3). The local tailoring of the fibre layer stiffness showed promise as a crack arresting feature, as can be seen in Figure 2.3 This initial investigation is the motivation for 
Glare $3-3 / 2-0.3$

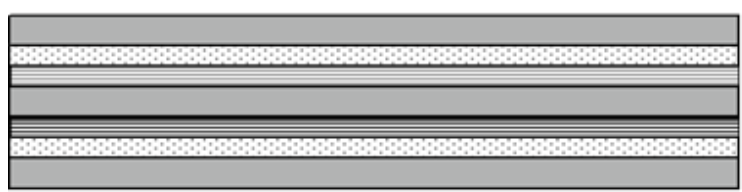

ITS Glare $3-3 / 2-0.3$

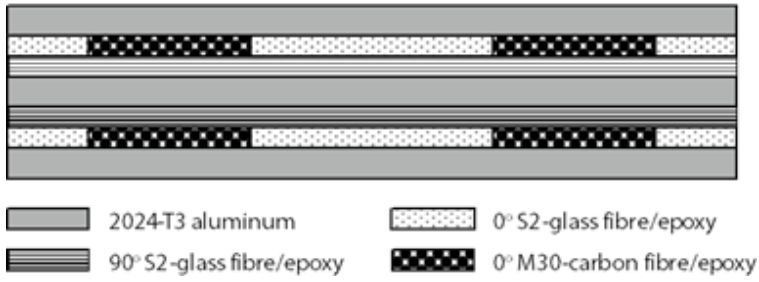

Figure 2.4: Internal tear strap in GLARE [16]

this thesis - to be able to accurately predict the fatigue crack growth rate within a variable stiffness fibre metal laminate.
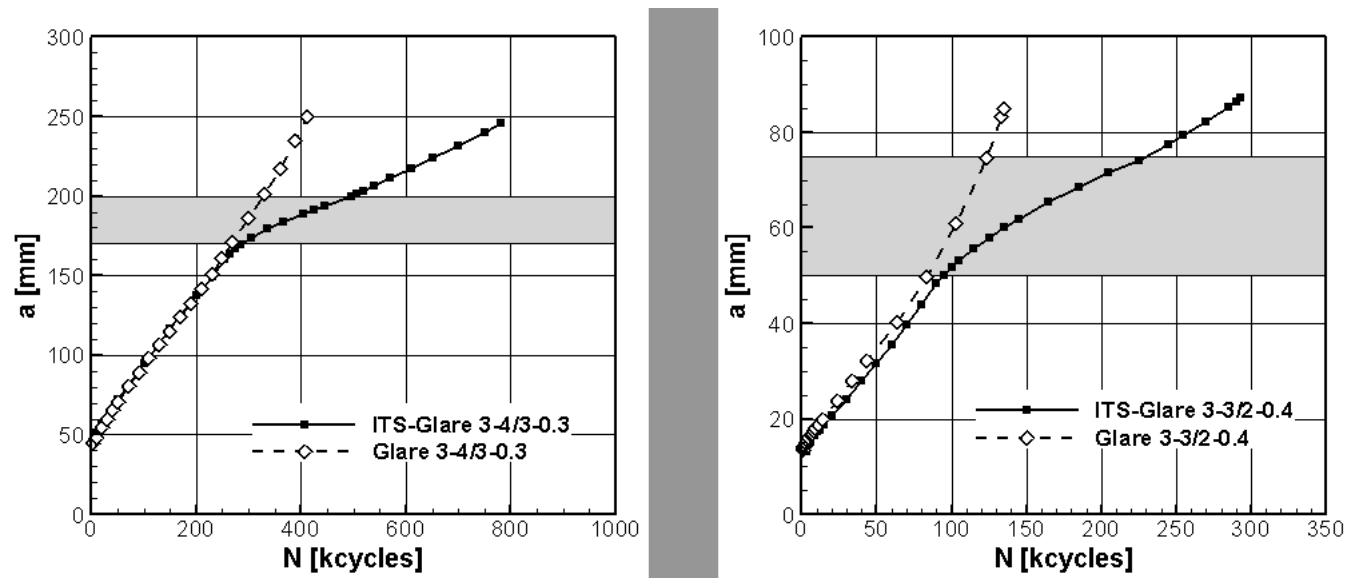

Figure 2.5: Effect of an internal tear strap on fatigue crack growth in GLARE [16]

To this effect, a baseline prediction methodology was chosen to begin the investigation. From the previous sections, it is clear that for the purpose of this investigation, a mechanistic model is preferable, as they are considerably more robust in terms of material and geometry configuration. As such, the Alderliesten model was selected as it has already been extensively validated and verified for a wide range of fibre metal laminate configurations [7]. 


\section{Chapter 3}

\section{Project Definition}

Previous models of fatigue crack growth in fibre metal laminates (FMLs) have proven successful in characterizing the behaviour of generic FMLs, however current models have been limited to uniform, symmetric laminates [7]. Some work was recently completed on characterizing fatigue performance of non-symmetric laminates [4].The goal of this thesis is to develop, validate and verify a simple analytical model for crack propagation and delamination growth within an FML with local stiffness variations, with principal focus on GLARE. The motivation for this research lies in expanding on research already carried out on local modification of the material composite type within a standard FML panel. It was found that this modification can act as a crack arresting feature [16]. The project investigated the impact on crack growth of two distinct cases:

- Examination of load redistribution as a result of stiffening elements present ahead of the crack tip.

- Changes in the bridging stress distribution due to stiffening elements behind the crack tip. 


\subsection{Project Scope}

In order to best study the effect of internal tear straps on fatigue crack growth in fibre metal laminates, a few restrictions have been placed on the materials and lay-ups that will be studied. These restrictions are laid out in the following sections.

\subsubsection{Modelling}

Focus within this project is given to variation of stiffness within the crack plane, in the direction perpendicular to the loading direction - similar to the application shown in Figure 2.3. This model will follow the formulation of Alderliesten's [7] model, based in linear elastic fracture mechanics. The crack growth rate is related to the stress intensity factor through an empirical Paris equation, as is the relation between the delamination growth rate and the strain energy release rate. The model will be limited to constant amplitude loading configurations, symmetric laminate configuration, and loading in the fibre direction.

\subsubsection{Materials}

For purposes of model verification and validation GLARE 2A - 2/1 - 0.3 was used in this investigation. GLARE $2 \mathrm{~A}$ is a symmetric lay-up, with 2 laminates of $0^{\circ}$ fibres for each fibre layer. Consequently this configuration has a high fibre bridging ability. The 2/1 - 0.3 layup has a nominal thickness of $0.866 \mathrm{~mm}$ which is very similar to the thickness of narrow-body aicraft fuselages constructed from monolithic aluminium.

A local variation in the fibre stiffness is achieved by substituting carbon fibre/epoxy for the glass fibre/epoxy in the loading direction. This application is not practical in 
aerospace applications due to the risk of galvanic corrosion, however the large stiffness ratio between the standard glass fibre used in GLARE and the carbon fibre presents a good study of the impact on fatigue life. As well, the previous investigations regarding local variations in stiffness in GLARE have made use of the same fibre combinations - expanding the available data sets for model verification [16].

While the testing programme will be restricted to these materials, the nature of the modelling process will allow for predictions of fatigue behaviour of other constituent materials, as the model's only inputs are material properties.

\subsubsection{Testing}

The fatigue crack growth tests were carried out under a constant amplitude with a peak stress of $140 \mathrm{MPa}$ and a stress ratio of 0.1 . Previous fatigue tests involving fibre metal laminates with local stiffness variations have been carried out with a peak stress of $120 \mathrm{MPa}$ and a stress ratio of 0.05 [16]. The rationale for the change in loading configuration was two-fold: the chosen panel configuration was significantly stiffer than those previously tested, leading to slower crack growth. Due to time constraints, the peak stress was increased. Secondly, the delamination growth constants used in modelling the Mode II delamination growth within the carbon fibre layers used were for a stress ratio of 0.1 , and as such the tests were carried out at a similar stress

ratio. Similarly to previous work done in validating the Alderliesten model, testing was carried out at $10 \mathrm{~Hz}[7]$. 


\section{Chapter 4}

\section{Implementation of the Modified Damage Growth Prediction Model}

\subsection{Introduction}

As laid out previously, modelling fatigue crack and delamination growth in fibre metal laminates is a particularly challenging task, ours made all the more challenging with the implementation of local variations in stiffness. The model which this investigation is based upon has certain limitations to reduce the complexity of the problem, namely that the fibre metal laminate be uniform and symmetric. As mentioned previously in Chapter 3, work has been done in modelling non-symmetric laminates [4], and this investigation seeks to allow for modelling of a variation in the stiffness of the fibre layers. The analytical model detailed in the following chapter will be limited to a stiffness variation parallel to the crack plane. The model is capable of accommodating functionally graded materials and is further limited to symmetric laminates. 
As this model is based on the work done previously by Alderliesten, the chief assumption present in this method is that Linear Elastic Fracture Mechanics are applicable [7]. Pursuant to this, the Paris relation between the effective change in stress intensity factors $\left(\Delta K_{e f f}\right)$ and the crack growth rate in the aluminium layers holds true, as does a similar relationship between the Strain Energy Release Rate (SERR) and the delamination growth rate in the fibre layers.

This model will employ a superposition of stress intensity factors in order to calculate the stress intensity factor at the crack tip, with $K_{t i p}$ in Equation 4.1 being evaluated as a difference between the far-field stress intensity factor $\left(K_{f f}\right)$ and the bridging stress intensity factor $\left(K_{b r}\right)$.

$$
K_{t i p}=K_{f f}-K_{b r}
$$

Considering the existing modelling methodology, the local variations in stiffness have two distinct effects: one ahead of the crack tip and one behind the crack tip (a local variation of stiffness at the crack tip can be divided into these two cases).

Section 4.3 covers the influence of a local stiffener ahead of the crack tip. Here an increased fibre stiffness draws load away from the damaged metal layer into the fibre layers. This effect is characterized by evaluating the far field Westergaard strain field and then applying strain compatibility through the thickness of the panel, resulting in a reduced $K_{f f}$.

Conversely, behind the crack tip, the stiffer fibres act to further reduce the crack opening displacement and as such increase the bridging stress intensity factor, reducing the effective stress intensity factor at the crack tip. Furthermore, the increased bridging load increases the SERR, resulting in accelerated delamination growth rates. These two effects are detailed in Section 4.4. 
Finally, due to the complex nature of the problem, a numerical implementation of this analytical model is performed, based in part on Alderliesten's previously published model [7], outlined in Section 2.2.2 and elaborated upon in the following section. This final implementation is discussed in Section 4.5.

\subsection{Numerical Implementation of the Alderliesten Crack Growth Model}

The fundamental concepts behind crack growth in FMLs, including the fibre bridging concept, were briefly described earlier in Section 2.2.2. Alderliesten's model for constant amplitude fatigue crack growth (developed for uniform, symmetric fibre metal laminates) shall form the basis of a prediction model for fibre metal laminates with local stiffness variations. A more detailed description of the implementation of the original model is therefore essential before the modifications can be detailed. For this investigation, the crack geometry (a), initial sawcut (s), delamination geometry (b), and the co-ordinate frame are illustrated in Figure 4.1. Note that this diagram represents the test specimens, not a real-world application (which would not contain a sawcut).

This model evaluates the reduction in stress intensity factor due to a reduction in crack opening displacement caused by intact fibres bridging the crack, as seen in Figure 4.2. This figure is representative of a generic damaged fibre metal laminate, showing the cracked metal layers, fibre layers, and the delamination shape.

In Adlerliesten's model, the bridging fibres cause the crack to close, resulting in a reduction in the stress intensity factor seen at the crack tip. This reduction in stress intensity factor is a function of the delamination geometry as well as the magnitude 


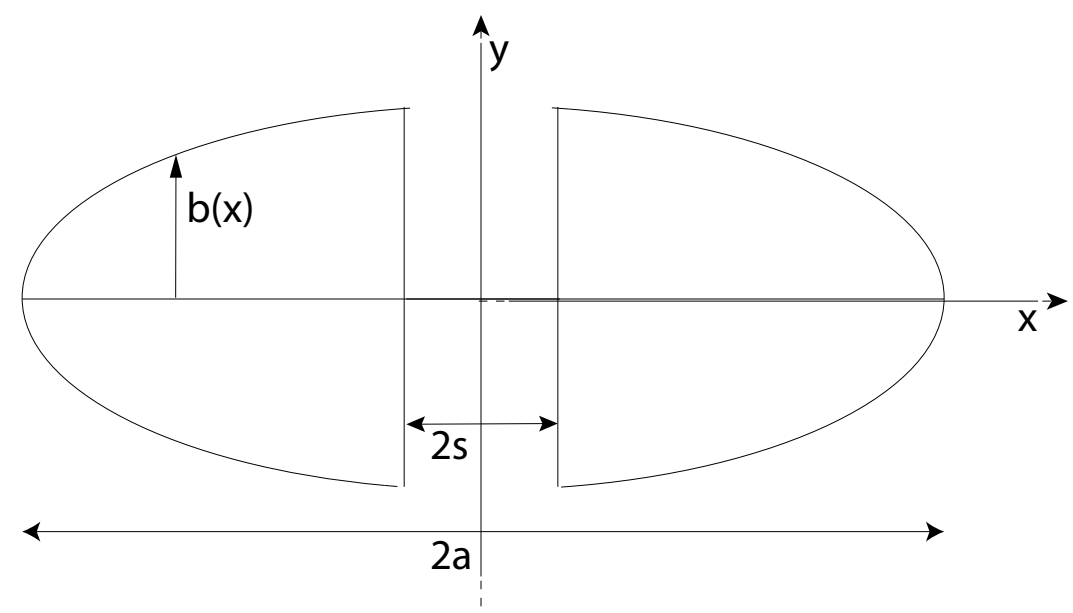

Figure 4.1: Generalized Crack and Delamination Geometry

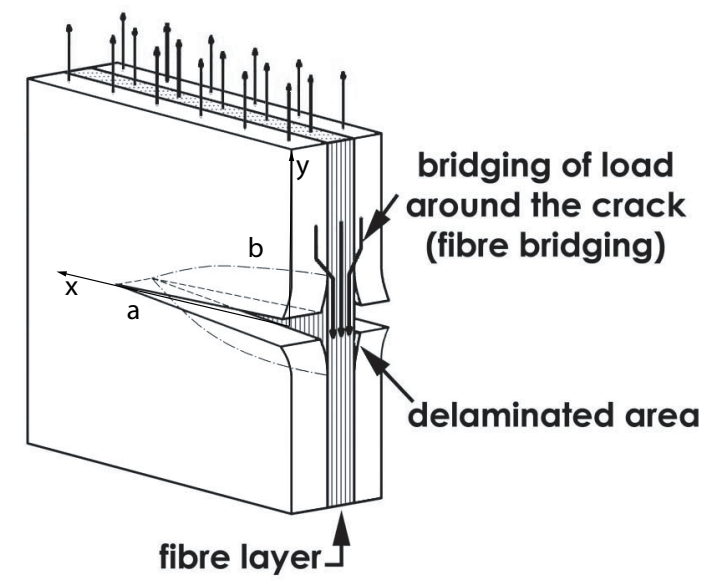

Figure 4.2: Fibre Bridging (Adapted from [7])

of the stress in the bridging fibres. The original model also predicts the growth rate of the delamination present behind the crack tip in the fibre layer. This growth rate is calculated based on the strain energy release rate present in the interface between the metal and fibre layers. Both of these growth rates are calculated using empirical Paris relations. 


\subsubsection{Paris Relations for Damage Growth Prediction}

Alderliesten's model uses Paris relations for predicting both the crack and interface delamination growth rates. Equation 4.2 is the basic Paris relation for fatigue crack growth in metal, with $C$ and $m$ being the empirical Paris constants for the metal being modelled.

$$
\frac{d a}{d N}=C\left(\Delta K_{e f f}\right)^{m}
$$

Before calculating the effective stress intensity factor at the crack tip, Alderliesten makes use of an empirical Dixon correction factor to account for finite-width geometries, presented in Equation 4.3, where $W$ is the panel width and $a$ is the crack length.

$$
\beta_{\text {Dixon }}=\frac{1}{\sqrt{1-\left(\frac{2 a}{W}\right)^{2}}}
$$

As the effective stress ratio $(R)$ at the crack tip is not constant, a relationship for $\Delta K_{\text {eff }}$ that includes a stress ratio correction is used. The particular stress ratio correction factor used in Alderliesten's model was derived by Schijve [17].

$$
\Delta K_{\text {eff }}=\beta_{\text {Dixon }}\left(1-R^{1.35}\right) K_{\text {max }}
$$

The Mode II Strain Energy Release Rate (SERR) at the delamination interface is defined as the change in strain energy for an increase in the delamination length under a given load. The stress used to evaluate this change in strain is a superposition of the far-field fibre stress and the bridging stress. The change in SERR $\left(\Delta G_{I I}\right)$ is calculated using the difference between the roots of the maximum and minimum SERR, as this 
relationship is most analogous to the change in stress intensity factor used in metal fatigue prediction [16].

$$
\frac{d b}{d N}=C_{b}\left(\Delta G_{I I}\right)^{m_{b}}=C_{b}\left(\sqrt{G_{I I_{\max }}}-\sqrt{G_{I I_{\min }}}\right)^{m_{b}}
$$

Here, $\frac{d b}{d N}$ is the delamination growth rate, $C_{b}$ is the empirical Paris coefficient, and $m_{b}$ is the empirical Paris exponent.

\subsubsection{Superposition of Far Field and Bridging}

As mentioned earlier in Equation 4.1, the stress intensity factor at the crack tip $\left(K_{t i p}\right)$ is calculated as a superposition of the far field stress intensity factor and the bridging stress intensity factor. The far-field stress intensity factor is derived from linear elastic fracture mechanics for a panel under uniform tension $\sigma_{f f, m}$ with a crack of length $a$.

$$
K_{f f}=\sigma_{f f, m} \sqrt{\pi a}
$$

The bridging stress intensity factor is calculated as a summation along the crack length of the stress intensity factor for a point load acting along each delamination width. These point loads cause the crack to close, and as such the bridging stress intensity factor reduces the stress intensity factor seen at the crack tip.

$$
K_{b r}=2 \sum_{i=1}^{N} \frac{\sigma_{b r, m}\left(x_{i}\right) w}{\sqrt{\pi a}}\left(1+\frac{0.5\left(1+\nu_{m}\right) b_{i}^{2}}{a^{2}-x_{i}^{2}+b_{i}^{2}}\right) \frac{a}{\sqrt{a^{2}-x_{i}^{2}+b_{i}^{2}}}
$$

The change in SERR evaluated using Equation 4.8 is used to determine the growth rate of the delamination using a Paris Relation laid out previously in Equation 
4.5 .

$$
G_{I I}(x)=\frac{M V F(x)\left(n_{f_{0}}+n_{f_{90}}\right) t_{f}\left(\sigma_{f}+\sigma_{b r, f}(x)\right)^{2}}{2 j E_{f}}
$$

The Metal Volume Fraction (MVF) defined in Equation 4.9 is used to calculate the portion of load that is transferred by the delamination interface.

$$
M V F(x)=\frac{n_{m} t_{m} E_{m}}{n_{m} t_{m} E_{m}+\left(n_{f 0}+n_{f 90}\right) t_{f} E_{f}}
$$

The number of delamination interfaces $(j)$ is determined by the number of metal layers, as seen in Equation 4.10.

$$
j=2\left(N_{m}-1\right)
$$

To solve for the bridging stress (which is needed for both the strain energy release rate and the stress intensity factor solutions), a displacement compatibility solution was derived.

\subsubsection{Displacement Compatibility}

The basis for the pre-existing model is displacement compatibility behind the crack tip between the delaminated fibre and cracked metal layers evaluated in the loading direction. This compatibility is summarized in Equation 4.11 and illustrated in Figure 4.3. The left hand side of the equation contains the metal layer contributions, with $v_{f f}$ representing the far-field crack opening displacement and $v_{b r}$ representing the reduction in crack opening due to bridging. The right hand side of the equation contains the contributions of the fibre layers, with $\delta_{p p}$ being the shear deformation of 
the prepreg adhesive, $\delta_{f f}$ being the far-field extension of the fibre layer, and $\delta_{b r}$ is the extension of the fibre layer due to the bridging load.
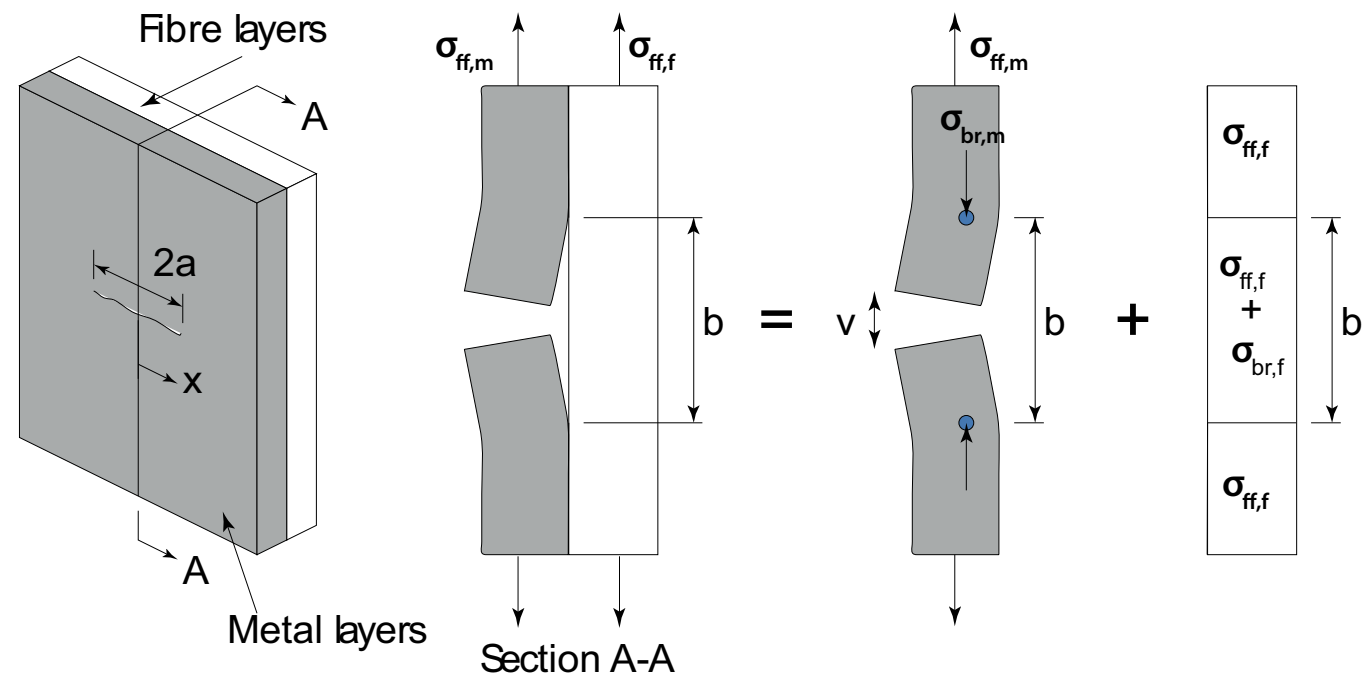

Figure 4.3: Displacement Compatibility (Modified from [16])

$$
v_{f f}-v_{b r}=\delta_{p p}+\delta_{f f}+\delta_{b r}
$$

\section{Crack Opening Displacement}

The left hand side of Equation 4.11 represents the total crack opening displacement. It is sub-divided into two cases: the crack opening in the metal layers due to the farfield metal layer stress $\left(v_{f f}\right)$, and the reduction in crack opening due to the transfer of farfield load into the fibre layer via the bridging stress $\left(v_{b r}\right)$.

Using linear elastic fracture mechanics, the crack tip opening displacement along the crack length direction, $\mathrm{x}$, can be expressed as a function of farfield load [18]. Written in terms of metal layer stresses and properties applicable to the problem at hand, this expression reduces to: 


$$
v_{f f}(x)=\frac{2 \sigma_{f f, m}}{E_{m}} \sqrt{a^{2}-x^{2}}
$$

Equation 4.13 represents the crack closing displacement due to the fibre bridging. The total bridging crack opening displacement is modelled as a series displacements caused by compressive point loads centred on each delamination zone, resulting in a reduction in net crack opening displacement.

$$
v_{b r}\left(x_{i}\right)=\int_{0}^{a} v\left(x_{i}, x_{j}\right) d x_{j}=\sum_{j=1}^{n} v\left(x_{i}, x_{j}\right)
$$

\section{Fibre Layer Deformation}

The fibre layer deformation is defined as the summation of the far field fibre $\left(\delta_{f f}\right)$, the prepreg shear $\left(\delta_{p p}\right)$, and the bridging fibre $\left(\delta_{b r}\right)$ deformations. The full derivation of these terms can be found in [7]. The first term, the fibre deformation, is derived from solid mechanics: it is the extension of the delaminated fibre lengths as a result

of the applied (far-field) and bridging stresses. The far-field stresses are known while the bridging stresses are unknown and must be solved for.

$$
\delta_{f f}(x)=\frac{\sigma_{f}+\sigma_{f_{b r}}(x)}{E_{f}} b(x)
$$

The shear prepreg deformation given in Equation 4.15 is also obtained from solid mechanics, with the shear stress across the delamination boundary defined in Equation 4.16 being a function of the delamination geometry, the load in the metal layer, and the interface compatibility. 


$$
\delta_{p p}(x)=\tau_{f}(x) \frac{\left(n_{f_{0}}+n_{f_{90}}\right) t_{f}}{G_{f}}
$$

$$
\tau_{f}(x)=\sigma_{f f, m} C_{b}(x) n_{m} t_{m} \sqrt{\frac{G_{f}}{\left(n_{f_{0}}+n_{f_{90}}\right) t_{f}}\left(\frac{1}{n_{m} t_{m} E_{m}}+\frac{1}{\left(n_{f 0}+n_{f 90}\right) t_{f} E_{f}}\right)}
$$

As the shear deformation is present in the delaminated fibre layer, it is dependent on delamination length and so Alderliesten introduced a correction factor $C_{b}$ to the shear stress at the delamination interface, shown in Equation 4.17.

$$
\begin{gathered}
C_{b}(x)=1-\left(\cosh \left(\sqrt{\alpha_{u d} b(x)}\right) \tanh \left(\sqrt{\alpha_{u d} b(x)}\right) \sinh \left(\sqrt{\alpha_{u d} b(x)}\right)\right) \\
\alpha_{u d}=\frac{G_{f}}{\left[\left(n_{f 0}+n_{f 90}\right) t_{f}\right]^{2} E_{f}}
\end{gathered}
$$

\section{Bridging Stress Solution}

Equation 4.19 contains the first part of the displacement compatibility equation a summation of all known displacements and elongations. In Alderliesten's model, this is modelled as a vector of displacements, with each displacement centered on a discrete delamination zone. This equation contains the prepreg shear deformation, the far-field crack opening displacement, and the far field fibre elongation.

$$
Q(i)=v_{f f}\left(x_{i}\right)-\delta_{p p}\left(x_{i}\right)-\frac{\sigma_{f}}{E_{f}} b\left(x_{i}\right)
$$

The $H$-matrix given in Equation 4.20 and represents the second (and final) part of 
the displacement compatibility equation. The $H$-matrix multiplied by the vector containing the bridging stresses gives the unknown bridging crack opening displacement and the bridging fibre elongation.

$$
H(i, j)=\frac{v\left(x_{i}, x_{J}\right)}{\sigma_{b r}\left(x_{j}\right)}+\frac{b(i)}{E_{f}} \delta(i, j)
$$

Equation 4.21 gives the final form of the displacement compatibility equation, setting the $Q$-vector equal to the $H$-matrix multiplied by the bridging stress vector. Solving the set of equations created by this formula gives the bridging stressed in the fibre layer.

$$
\{Q\}=[H]\left\{\sigma_{b r, f}\right\}
$$

The final step before returning to the superposition step in Alderliesten's model is to evaluate the bridging stresses in the metal layer - these are calculated through the thickness ratio between the fibre and metal layers.

$$
\sigma_{b r, m}\left(x_{j}\right)=\frac{\sigma_{b r, f}\left(x_{j}\right)\left(n_{f_{0}}+n_{f_{90}}\right) t_{f}}{n_{m} t_{m}}
$$

Now that Alderliesten's original model has been outlined, the modificatons made to correct for variations in stiffness behind and ahead of the crack tip can be implemented. 


\subsection{Implementation of Variation of Fibre Stiffness Ahead of Crack Tip}

As mentioned earlier, the presence of a variation in stiffness ahead of the crack tip will result in a redistribution of the load throughout the structure. An increase in stiffness ahead of the crack tip will draw load away from the crack tip and reduce the stress intensity factor at the crack tip. This section details the tools used to model the redistribution of load from the crack tip to the stiffened fibre sections.

This approach makes use of strain compatibility through the thickness of the panel. The strain is equal through the thickness of the panel and as such, the stress in each layer of the panel can be calculated using stiffness ratios.

The first step in modelling the load redistribution ahead of the crack tip is to evaluate the stress in the intact metal layer. This stress is calculated from the Westergaard [18] stress solution for a finite width panel under uniform tension $\sigma_{f f, m}$ and crack halflength of $a$.

$$
\sigma_{y y, m}(x)=\frac{\sigma_{f f, m}}{\sqrt{1-\frac{a^{2}}{x^{2}}}}
$$

The next step is to evaluate the stress in the fibre layer relative to the metal layer. Using strain compatibility and modelling the fibre layers using a piecewise continuous function, gives the relationship shown in Equation 4.24.

$$
\sigma_{y y, f}(x)=\frac{E_{f}(x)}{E_{m}} \frac{\sigma_{f f, m}}{\sqrt{1-\frac{a^{2}}{x^{2}}}}
$$

The penultimate step to solving for the redistribution ahead of the crack tip is to 
integrate the stress in each layer multiplied by its respective thickness from the crack tip to the panel edge. The summation of these integrals is equal to the load present in the panel ahead of the crack tip. Rearranging the integration expression, we can solve for the far field stress in the metal layer, as shown in Equation 4.25.

$$
\sigma_{f f, m}=\frac{P_{t i p}}{\int_{a}^{\frac{W}{2}}\left[n_{m} t_{m}+\left(n_{f 0}+n_{f 90}\right) t_{f} E_{f}(x)\right] \frac{1}{\sqrt{1-\frac{a^{2}}{x^{2}}}} d x}
$$

The load ahead of the crack tip $\left(P_{\text {tip }}\right)$ can be determined from the total load $\left(P_{\text {total }}\right)$ on the panel by subtracting the load carried by the fibres behind the crack tip.

$$
P_{\text {tip }}=P_{\text {total }}-\sum_{i=1}^{N} \sigma_{f f, f}\left(x_{i}\right) w\left(x_{i}\right)\left(n_{f 0}+n_{f 90}\right) t_{f}
$$

This updated far field metal layer stress is then used in Equation 4.6 to calculate the far field stress intensity factor in the original model.

\subsection{Implementation of Variation of Fibre Stiffness Behind Crack Tip}

There were several further modifications made to Alderliesten's original model to accommodate variations in stiffness behind the crack tip. The following modifications were made to accommodate for changes in stiffness (or fibre material) behind the crack tip: the far-field crack opening displacement, the delamination growth rate calculation, and the fibre stress calculation.

The far-field crack opening displacement laid out in Alderliesten's original model and 
seen earlier in Equation 4.12 is updated to correspond with the stress solution given by Westergaard [18]. The updated crack opening displacement shown in Equation 4.27 includes a correction factor for Poisson effects.

$$
v_{f f}(x)=\frac{2\left(1-\nu^{2}\right)}{E} \sqrt{a^{2}-x^{2}}
$$

The next correction to the original algorithm was made to the determination of the SERR - the calculation of the Metal Volume Fraction was changed to accommodate a change in stiffness of the fibre layer. Previously, the MVF calculation generated a single value which was then used in the calculation of the vector containing the SERR for each finite delamination width. The updated calculation creates a vector of MVF values, one for each finite delamination width, weighted by the fibre type contained within the delamination width.

Finally, any calculation that made use of a constant fibre stiffness or other material properties (namely Equations 4.8, 4.14, and 4.20) were changed to take into account a continuous function for fibre stiffness or the respective property.

\subsection{Overall Model Implementation}

The final model was then implemented in the MATLAB environment with Figure 4.4 highlighting the process flow. To begin with, the simulation takes in the test parameters (the peak load, stress ratio, operating temperature, and maximum number of cycles) and the model inputs (specimen geometry, fibre types and their respective material properties, metal properties, paris relations for crack and delamination growth, specimen cure temperature, and initial crack size). 


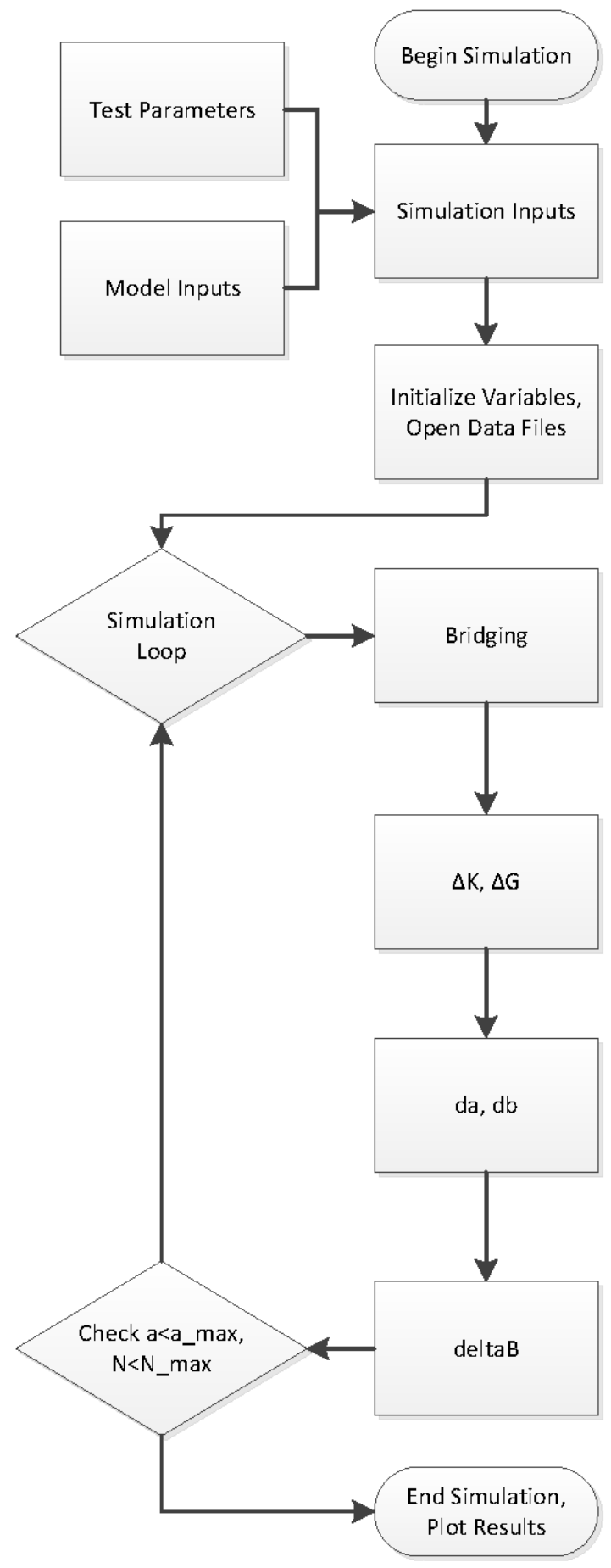

Figure 4.4: Model Implementation - Simulation Flow 


\section{Model Initialization}

Following this, a calculation of the applied and residual stresses in each layer and section is performed. In order to do this, a generalized stiffness is defined for the structure components. For any $n$ layers of section $i$ of the laminate with Young's Modulus $(E)$, width $(W)$, and thickness $(t)$ the effective stiffness defined in Equation 4.28 is used.

$$
k_{i}=\frac{E_{i} W_{i} t_{i} n_{i}}{L_{i}}
$$

The total stiffness of the laminate is then given by the summation of these individual generalized stiffnesses.

$$
k_{\text {lam }}=\sum_{i=1}^{n} k_{i}
$$

As fiber metal laminates are cured in autoclave, and as the materials involved have differing coefficients of thermal expansion, there are residual mechanical strains present in the room temperature laminate. To calculate the residual curing stresses within the laminate, the forces in all layers of the laminate are assumed to be in equilibrium, each layer has the same initial length, and assuming strain compatibility between the layers, the relationship in Equation 4.30 can be used.

$$
\sum_{i=1}^{n} k_{i} \delta_{i}=0
$$

For a generic panel, $\delta_{i}$ is the total elongation of the panel and from this the total strain within a section $i$ of the panel can then be calculated by the ratio of the elongation and the initial length. This total strain in each layer must be equal to superposition 
of the thermal strain and the mechanical strain.

$$
\epsilon_{i}=\frac{\delta_{i}}{L_{i}}=\epsilon_{l a m}-\alpha_{i} \Delta T
$$

Combining Equations 4.30 and 4.31 and solving for the mechanical strain present in each layer results in Equation 4.32. Using this, for a layer $i$, the residual strain can now be calculated.

$$
\begin{gathered}
\epsilon_{i}=\frac{\sum_{j=1}^{n} k_{j}\left(\alpha_{j}-\alpha_{i}\right)}{k_{l} a m} \Delta T \\
\sigma_{i_{\text {res }}}=\epsilon_{i} E_{i}
\end{gathered}
$$

From this mechanical strain, we can use solid mechanics to calculate the residual stress present in each section of the structure. The next step follows the same process, however determines the respective far field stress in each section of the structure based on an applied stress. The applied far field stress within a section $i$ of a panel of width $W$ and thickness $t$ is given in Equation 4.34.

$$
\sigma_{i_{a p p}}=\sigma_{f f} \frac{k_{i}}{k_{\text {lam }}} \frac{W t}{W_{i} t_{i} n_{i}}
$$

The total stress within each section is then calculated as a superposition of the applied and residual stresses. This calculation is performed for both the maximum and minimum loads the panel is subjected to.

The delamination zones are then initiated, with a minor change to the finite widths defined by Alderliesten. The original model has equal delamination widths. The 
closer a bridging element is to the crack tip the greater its respective contribution to the bridging stress intensity factor, a new delamination width definition has been developed. It makes use of a Cehbyshev relation, which biases the delaminations distribution to the crack tip - that is, the closer to the crack tip, the more delamination zones are present within a fixed distance. This modification has been found to improve the bridging stress resolution [4].

Following the initiation of the delamination widths, the initial delamination lengths are defined. Alderliesten defined multiple initial delamination shapes, however found that the initial shape of the delamination had little effect on the final shape after several simulation loops [7]. As such, the elliptical definition described in Equation 4.35 is used to initialize the delamination lengths.

$$
b(x)=0.8 \sqrt{1-\left(\frac{x-s}{a-s}\right)^{2}}
$$

The simulation loop is then initialized and the following subfunctions are evaluated: Bridging, $\Delta K, \Delta G$, da/db, deltaB. The details of these specific functions follow.

\section{Module: Bridging}

This function calculates the bridging stress distribution, the effective stress intensity factor and the strain energy release rate for a given loading case, delamination geometry, and crack length.

The function begins by evaluating the stress at the crack tip, making use of the previously described methods to correct for the variation in stiffness ahead of the crack tip. This new stress is used in the application of Alderliestens displacement compatibility equation to determine the bridging stress distribution along the crack 
length. This bridging stress is then used to calculate the bridging stress intensity factor. The effective stress intensity factor is then calculated as the superposition of the far-field stress intensity factor and this bridging stress intensity factor. The function subsequently calculates the strain energy release rate using the far field fibre stress and the bridging fibre stress and passes these values to the next module.

Module: $\Delta K, \Delta G$

This function calculates the change in stress intensity factor as well as the change in strain energy release rate given peak/valley values for each. The stress intensity formulation includes a correction for R-ratio effects from Schijve [17] to obtain $\Delta K_{\text {eff }}$. The updated correction factor for the effective stress intensity factor is given in Equation 4.37. Note that this model does not use the empirical Dixon correction factor as finite edge effects are accounted for in the Westergaard stress solution used in the load redistribution section.

$$
\begin{gathered}
R_{t i p}=\frac{K_{t i p_{\max }}}{K_{t i p_{\min }}} \\
\Delta K_{\text {eff }}=\left(0.55+0.33 R_{t i p}+0.12 R_{\text {tip }}^{2}\right)\left(1-R_{t i p}\right) K_{\text {max }}
\end{gathered}
$$

\section{Module: $\mathrm{da} / \mathrm{db}$}

This function calculates the crack and delamination increments. The function makes use of $\Delta K_{\text {eff }}$ and the Paris relation to determine the number of cycles required to grow the crack a fixed length. This number of cycles is then used in coordination with the Paris relation and the calculated SERR for the delamination growth rate to 
calculate the delamination increments.

\section{Module: deltaB}

This function updates the delamination geometry by using the old delamination geometry (including elements widths and locations), the vector containing the delamination increments, and the new crack length then uses a Piecewise Cubic Hermite Interpolating Polynomial (pchip) to fit a new curve to an updated set of delamination lengths and widths.

Once these four modules have completed, the simulation checks the total number of cycles, the current crack length and if the two values are under their maximum values, restarts the loop. Once either value exceeds its respective limit, the simulation terminates and outputs the results to file. 


\section{Chapter 5}

\section{Model Validation and Verification}

\subsection{Introduction}

"Validation is the process of determining the degree to which a model, simulation, or federation of models and simulations, and their associated data are accurate representations of the real world from the perspective of the intended use(s).

Verification is the process of determining that a computer model, simulation, or federation of models and simulations implementations and their associated data accurately represent the developer's conceptual description and specifications." [19]

As laid out in Chapters 2 and 4, modelling fatigue crack growth in fibre metal laminates is a challenging task. Due to the complexity of the model described in Chapter 4, multiple steps are taken to validate and then to verify the model.

The first section of this chapter covers validation of the fatigue crack growth model first it confirms fidelity between the new and the original models and then validates the load redistribution model. Following this, verification of the overall model predictions 
for the two principal fatigue damage types: crack propagation and delamination growth.

\subsection{Testing and Simulation Parameters}

Prior to laying out the validation and verification of the implemented model, the relevant simulation and testing parameters should be detailed. They are found following, in Table 5.1. A complete list of material properties can be found in Appendix A.

Table 5.1: Model Inputs and Testing Parameters

\begin{tabular}{ccc}
\hline Symbol & Description & Value \\
\hline$C$ & Paris coefficient - aluminium & $3.69 \mathrm{E}-13$ \\
$m$ & Paris exponent - aluminium & 3.591 \\
$C_{b}$ & Paris coefficient - glass fibre/epoxy & 0.05 \\
$m_{b}$ & Paris exponent - glass fibre/epoxy & 7.5 \\
$C_{b_{c}}$ & Paris coefficient - carbon fibre/epoxy & 0.0943 \\
$m_{b_{c}}$ & Paris exponent - carbon fibre/epoxy & 5.8648 \\
$m_{a x w}$ & Maximum delamination zone width & $0.05 \mathrm{~mm}$ \\
$d a$ & Crack length increment & $0.01 \mathrm{~mm}$ \\
$f$ & Testing frequency & $10 \mathrm{~Hz}$ \\
\hline
\end{tabular}

\subsection{Comparison of updated model to original Alderliesten model}

As this model is based on a pre-existing model, confirmation of the new model's fidelity to the original one is needed before proceeding with validation of the load 
redistribution mechanism. Validation of the new model with respect to the original Alderliesten model will take place in three parts: first, validation of the crack opening displacement regime, followed by validation of the predicted fatigue crack growth rates and delamination shapes.

As laid out in Chapter 4, the Alderliesten fatigue crack growth model's fundamental basis is the displacement compatibility through the crack interface. As such, validation of the new model's fidelity to the original model's crack opening displacement predictions will confirm the new model's fidelity in crack growth prediction. The motivation for both the Alderliesten and this new model is to be able to accurately predict the life of parts in service - as such accurate fatigue crack growth and delamination shapes are key to validation of this model. First validating the model's behaviour and fidelity to the Alderliesten model will allow us to determine which shortcomings are as a result of the Alderliesten base model and which have been caused by the implementation laid out in Section 4.3.

\subsubsection{Crack opening displacement validation}

The first validation test is a comparison of the crack opening displacement predicted by this investigation, the prediction of Alderliesten's model, and test results sourced from Alderliesten [7]. Confirmation that the model described in Chapter 4 maintains fidelity with the Alderliesten model should confirm that the displacement compatibility condition behind the crack tip is maintained. Figure 5.1 shows good correlation between both the prediction and the test results as well as between the prediction and the original Alderliesten model predictions. As this investigation's model is based on crack opening relations, these results increase confidence in the overall model.

As the model is more sensitive to changes in crack opening displacement closer to 


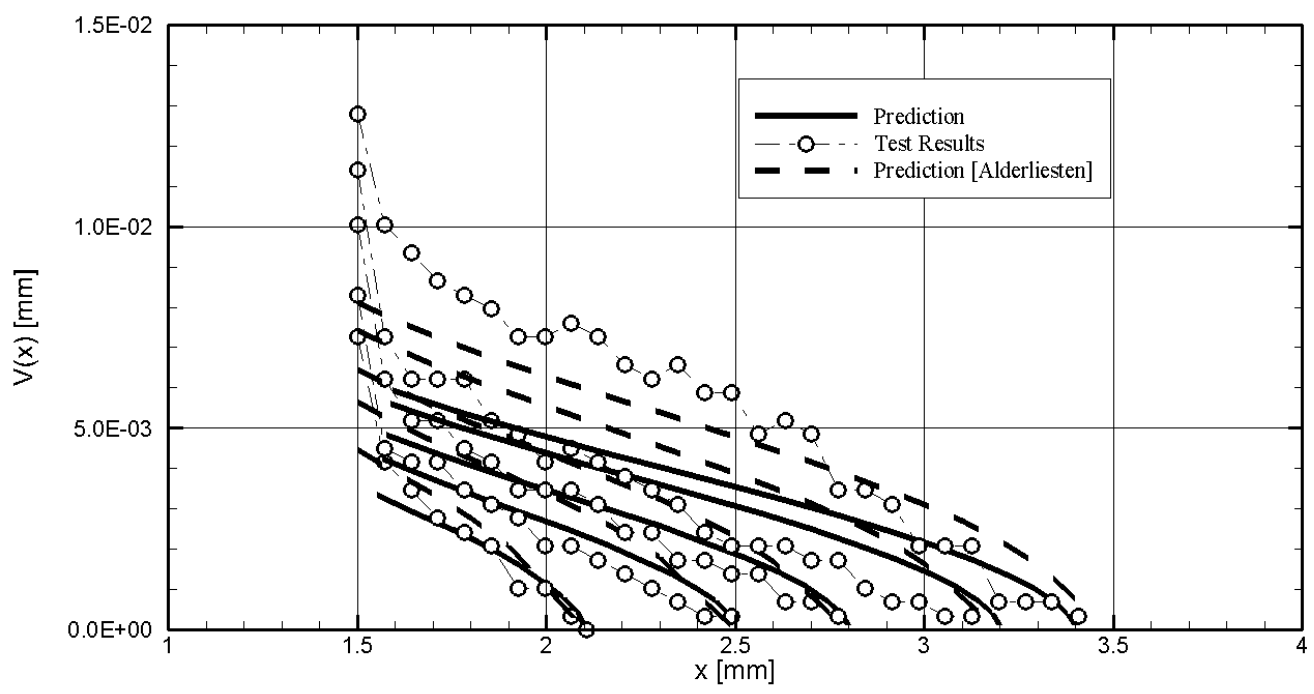

Figure 5.1: Comparison between predicted (original Alderliesten model and updated model) and measured crack opening of GLARE 3-6/5-0.4 with $W=100 \mathrm{~mm}, \sigma_{\max }=$ $100 M P a, R=0.05,2 a_{0}=3 \mathrm{~mm}$. Constructed with test data from [7].

the crack tip and less so to variations further from the crack tip, better correlation at the crack tip is preferred - which this validation test confirms is the case. A note should be made that significant deviation between the predicted and measured crack opening displacement occurs at the sawcut tip $(x=1.5 \mathrm{~mm}$ in Figure 5.1) as this is a free edge in the test specimens.

\subsubsection{Fatigue crack growth model validation}

For the purposes of validating the crack opening model implemetation, comparison to three cases from Alderliesten's model verification process [7] will be used. The first two cases presented in Figures 5.2 and 5.3 consist of GLARE 3 in a $4 / 3$ configuration using $0.5 \mathrm{~mm}$ thickness aluminium sheets.

Figure 5.2 consisted of a $500 \mathrm{~mm}$ wide panel with a maximum load of $100 \mathrm{MPa}$ and a stress ratio of 0.05 . The model prediction shows good correlation with both the test 


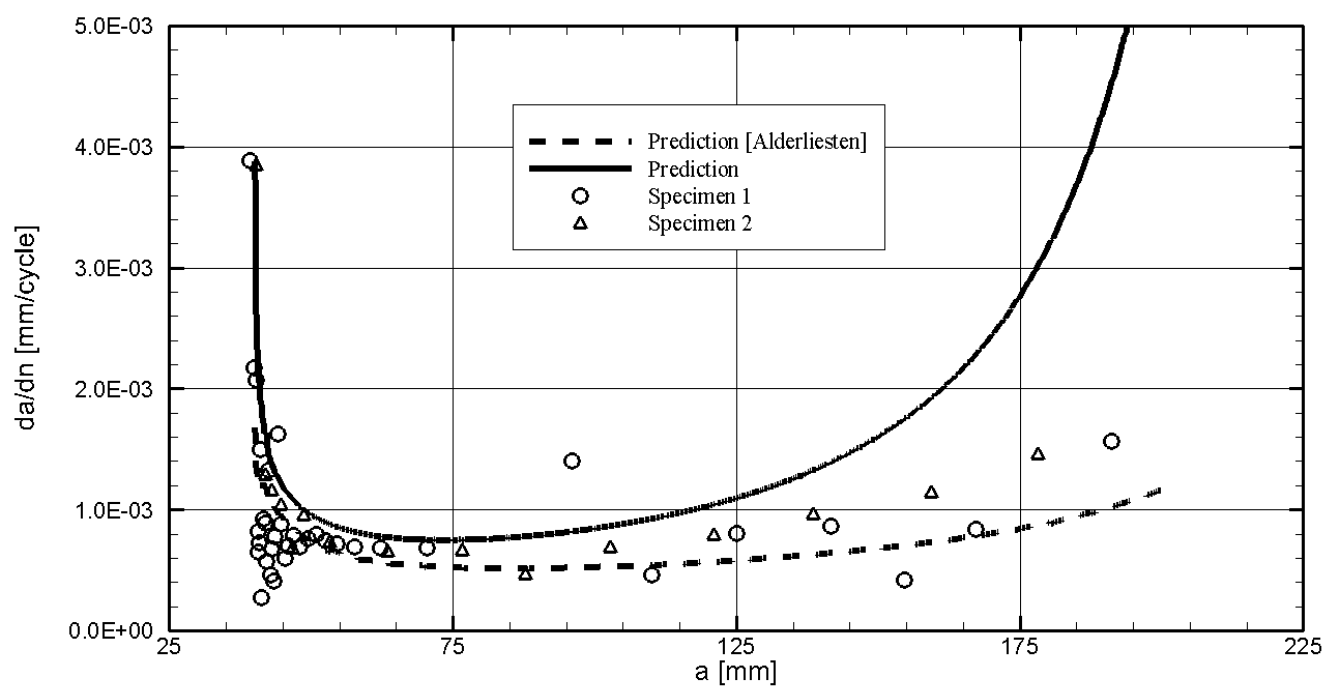

Figure 5.2: Comparison between predicted (original Alderliesten model and updated model) and crack growth rates for GLARE 3-4/3-0.5 with $W=500 \mathrm{~mm}, \sigma_{\max }=$ $100 M P a, R=0.05,2 a_{0}=75 \mathrm{~mm}$. Constructed with test data from [7].

results and Alderliesten's original model predictions. These panels were wide-panel tests, meaning that the crack grew very close to the edge of the panel. Herein can be seen the first major departure of this investigation's model from the Alderliesten model. As the crack approaches the free edge of the panel, the two different models diverge greatly.

The cause of the divergence as the crack-to-width ratio approaches unity lies in each model's approach to correcting for finite-width geometries. The Alderliesten model uses the Dixon empirical correction factor to account for these geometries whereas the model presented herein accounts for these geometries through the Westergaard stress function. The result of using the Westergaard is that the newer model then overpredicts the fatigue crack growth rate for larger crack-to-width ratios. This deficiency bears further study, yet lies outside the scope of this study. Note that in actual application in an aerospace setting, it is very unlikely that a crack will grow to such a large size without drawing an inspector's attention. 


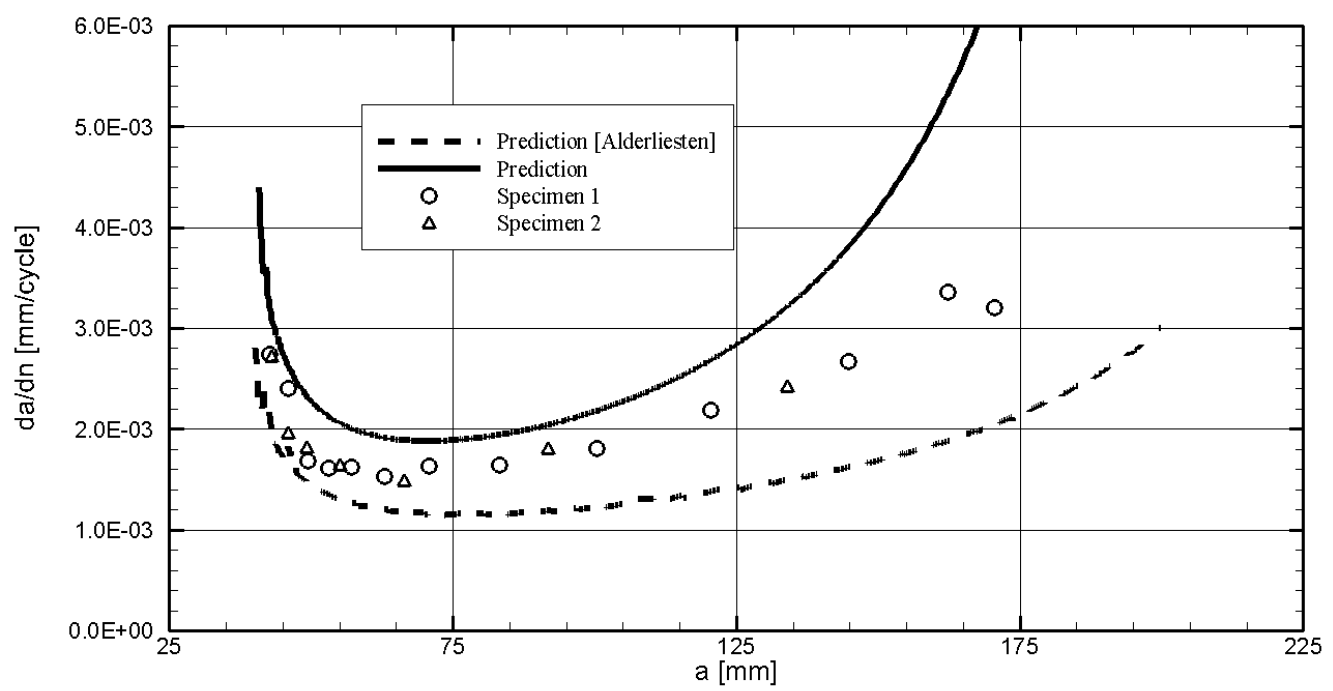

Figure 5.3: Comparison between predicted (original Alderliesten model and updated model) and crack growth rates for GLARE 3-4/3-0.5 with $W=500 \mathrm{~mm}, \sigma_{\max }=$ $120 M P a, R=0.05,2 a_{0}=75 \mathrm{~mm}$. Constructed with test data from [7].

The next comparison, Figure 5.3 has the same configuration as Figure 5.2, and was tested at a peak load of $120 \mathrm{MPa}$ with a stress ratio of 0.05 . The same observations can be made for this test case as with the previous one - that the new model slightly overpredicts crack growth rates initially, then deviates greatly as the crack-to-width ratio approaches unity. The difference is slightly magnified, however this is explained simply enough by the higher peak load.

The final crack growth comparison test case presented here is for a narrow panel the fatigue crack was not grown as close to the panel edge as it was in the previous two examples. With this test case, it is clearly recognizable that the new model holds true to the Alderliesten model - a very slight deviation in crack growth rate is seen at the onset, but once the crack growth rate stabilizes, the two models are very close to each other as well as to the recorded test data. In this test case, the deviation seen in the previous two figures is not observed here as a result of the smaller crack-to-width ratio. 


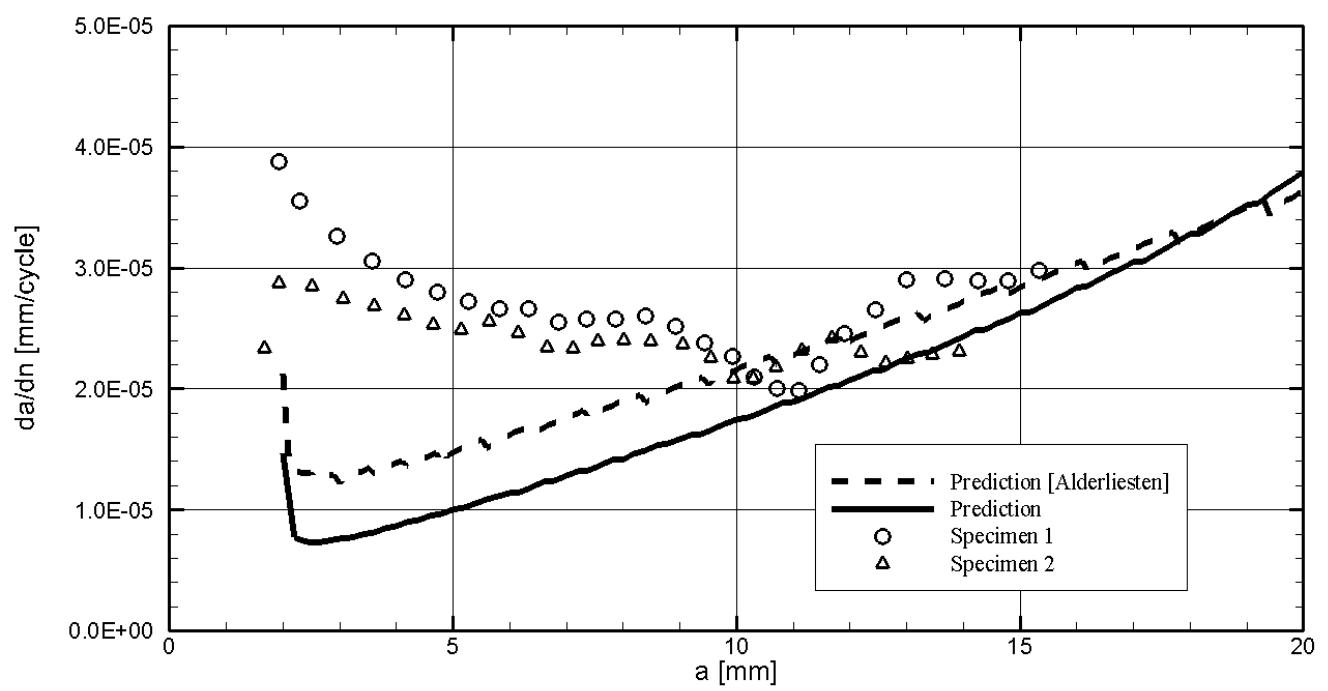

Figure 5.4: Comparison between predicted (original Alderliesten model and updated model) and crack growth rates for GLARE 3-6/5-0.4 with $W=100 \mathrm{~mm}, \sigma_{\max }=$ $100 \mathrm{MPa}, R=0.05,2 a_{0}=3 \mathrm{~mm}$. Constructed with test data from [7].

\subsubsection{Delamination shape validation}

For the purposes of validating the new model against the Alderliesten model, one final test is performed - a check of the predicted delamination shape. As the model shows good fidelity against the Alderliesten model in crack opening shape and fatigue crack growth rate predictions, good delamination shape correlation will further confirm the model's validity. The test case selected for Figure 5.5 is the same as the one for Figure 5.3.

The predicted delamination shapes correspond well with the original Alderliesten predictions - very little variance is seen between them. The new model exhibits the same limitations as the Alderliesten model - an underpredicting of the delamination shape far away from the crack tip. Again, as the bridging load closer to the crack tip has a larger impact on the bridging stress intensity factor, this difference is not too concerning. Furthermore, this difference is caused by the nature of the model 


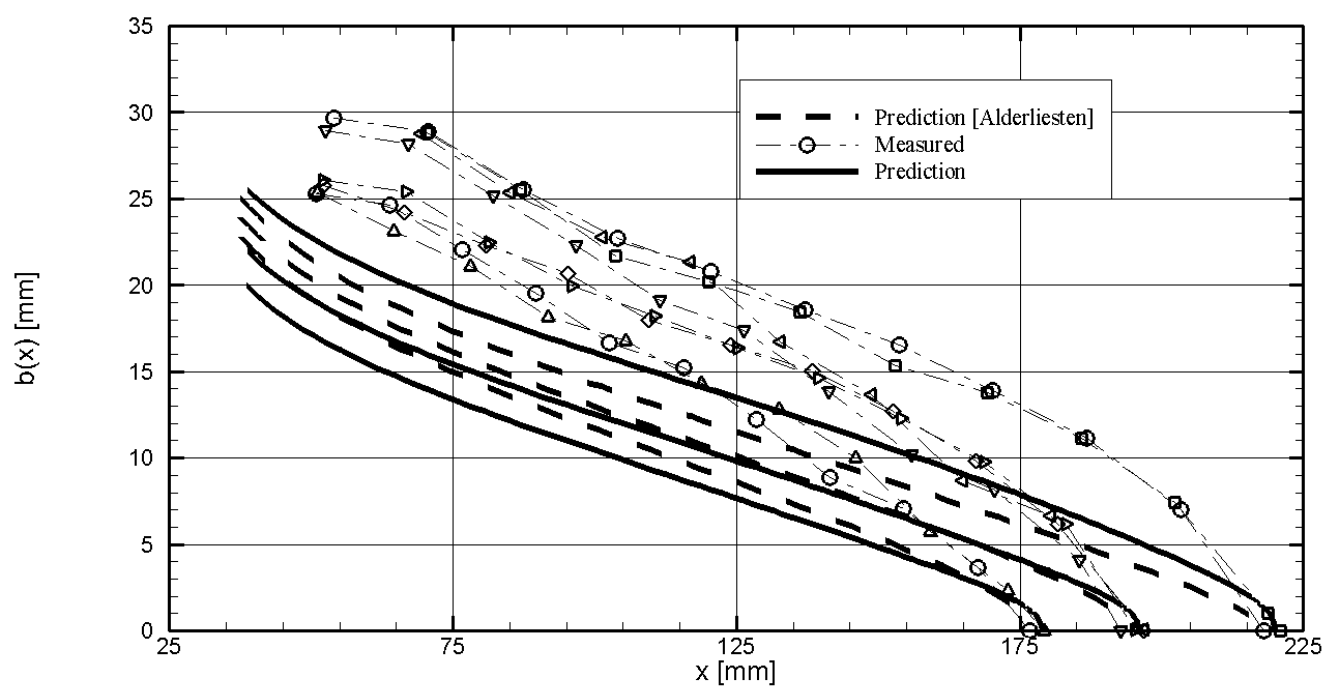

Figure 5.5: Comparison between measured and predicted (Alderliesten and updated model) delamination sizes for GLARE 3-4/3-0.5 with $W=500 \mathrm{~mm}, \sigma_{\max }=120 \mathrm{MPa}$, $R=0.05,2 a_{0}=75 \mathrm{~mm}$. Constructed with test data from [7].

implementation - as it consists of multiply-calculated growth intervals, there exist cumulative errors the more cycles required to achieve the required crack length. A small difference in the predicted delamination growth rate (from either the model or from the empirical constants used) can result in large differences in delamination shapes.

\subsection{Validation of load redistribution model}

As the load redistribution model is the main modification made to the Alderliesten fatigue crack growth model, validation of the methodology involved is key to overall validation the updated model. This section will focus on validation of the stress field predictions ahead of the crack tip and the bridging stress predictions behind the crack tip. To validate these results, an in-situ measurement methodology is preferred, as this allows for multiple measurements from a single test specimen. The selected 
methodology, Digital Image Correlation (DIC), has been used sucessfully in the past to measure strain fields in fibre metal laminates [20].

In preparing for DIC, the test specimens have a fine high-contrast speckle coat applied. To evaluate the strain field within a test specimen, two reference images are taken - one each of the maximum and minimum loading configurations. These images are then discretized into small zones, each containing a set of black and white pixels (the result of the speckle pattern). The processing algorithm (implemented by Vic3D ${ }^{\mathrm{TM}}{ }_{\text {system }}$ offered by Correlated Solutions) determines the relative displacement and deformation of the pixel groups between the two reference images to calculate relative strains. These strains are then compared to the prediction results for the relevant crack and delamination geometry.

Two test specimens were created for the purposed of validating the load redistribution model. The test specimens consisted of inverted GLARE 2A lay-ups with an adhesive layer (with nylon carrier) bonding the two metal layers. Each specimen contained a transition from glass/epoxy to carbon/epoxy or vice versa in order to see the effect of a change in stiffness on the strain distribution. An inverted layup is used so that the fibre layers are on the exterior of the panel, allowing direct measurement of fibre strains using the DIC method. A consequence of this layup, however, is that the aluminium layers are hidden, and crack growth measurements cannot be directly made. See Appendix B for further information on the specimen configuration and preparation.

Figures 5.6 and 5.8 show the comparison between measured and predicted fibre layer strains for a Carbon-Glass-Carbon configuration. The first of these, Figure 5.6, acts as a check for the first of two major cases: a variation and increase in fibre stiffness ahead of the crack tip. Overall, good correspondence is achieved between the measured and predicted fibre layer strains. The model slightly underpredicts the bridging load 


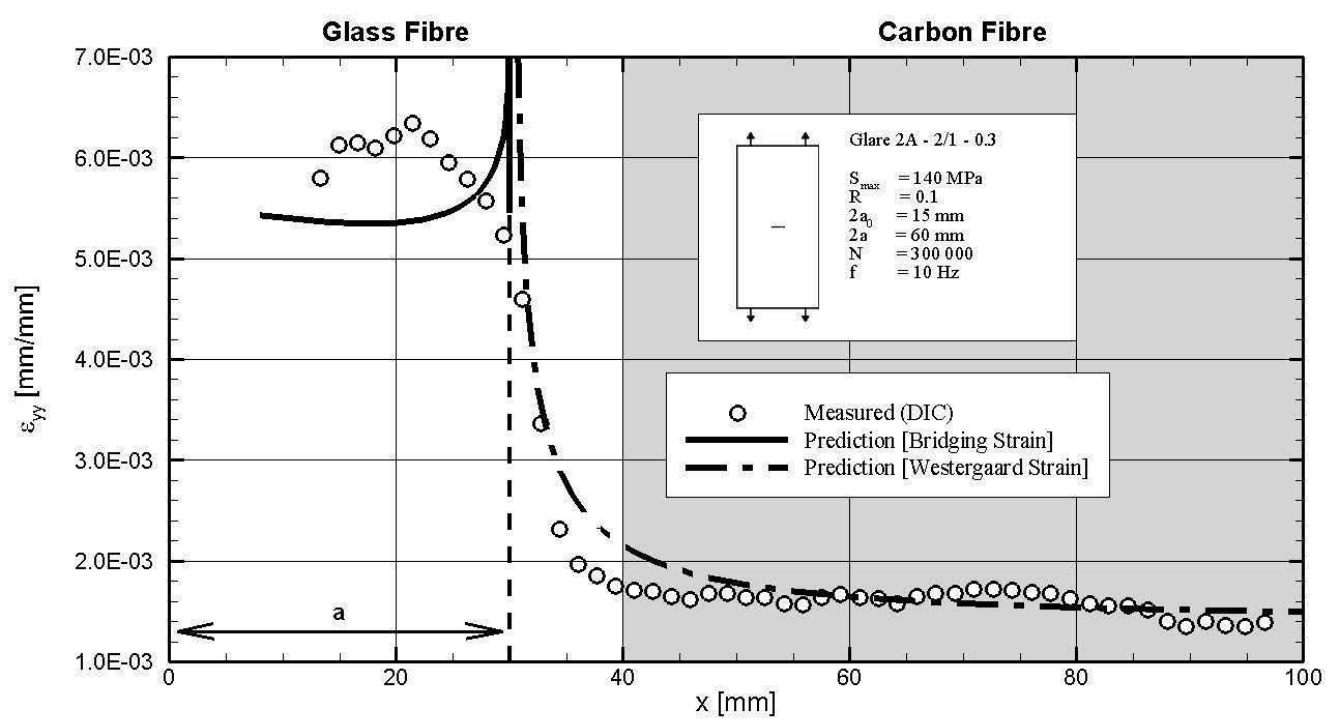

Figure 5.6: Comparison of predicted and measured fibre layer strains for CarbonGlass-Carbon configuration ITS-GLARE 2A-2/1-0.3 with $W=85 / 80 / 85 m m, \sigma_{\max }=$ $140 M P a, R=0.1,2 a_{0}=15 \mathrm{~mm}, N=300000$.

further from the crack tip, however this underprediction is offset by the linear elastic nature of the model. As the model does not take into account plasticity and plastic zone size, the predicted strains asymptotically trend towards infinity both behind and ahead of the crack tip.

The model's ability to model the second major case, a decrease in fibre stiffness ahead of the crack tip, is presented in Figure 5.7. There is good agreement between the predicted and measured strains, once again confirming the model's ability to accurately predict the fibre layer strains.

Figure 5.8 is included as a robustness check to validate the model's performance in predicting the load redistribution as the crack grows through a material transition. Again, good correspondence between the predicted and measured fibre layer strains is seen, with deviation at the crack tip.

As all of the above comparison tests show good agreement between the predicted and 


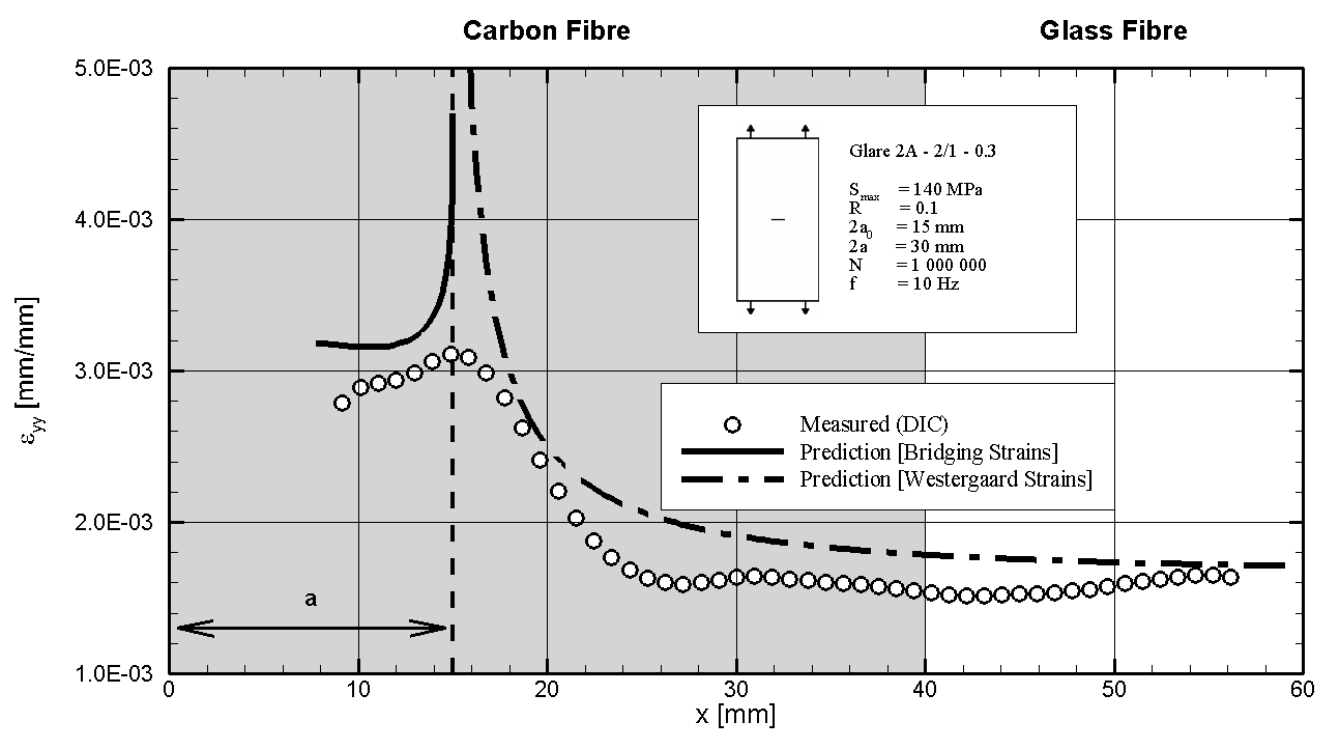

Figure 5.7: Comparison of predicted and measured fibre layer strains for GlassCarbon-Glass configuration ITS-GLARE 2A-2/1-0.3 with $W=85 / 80 / 85 m m, \sigma_{\max }=$ $140 M P a, R=0.1,2 a_{0}=15 \mathrm{~mm}, N=1000000$.

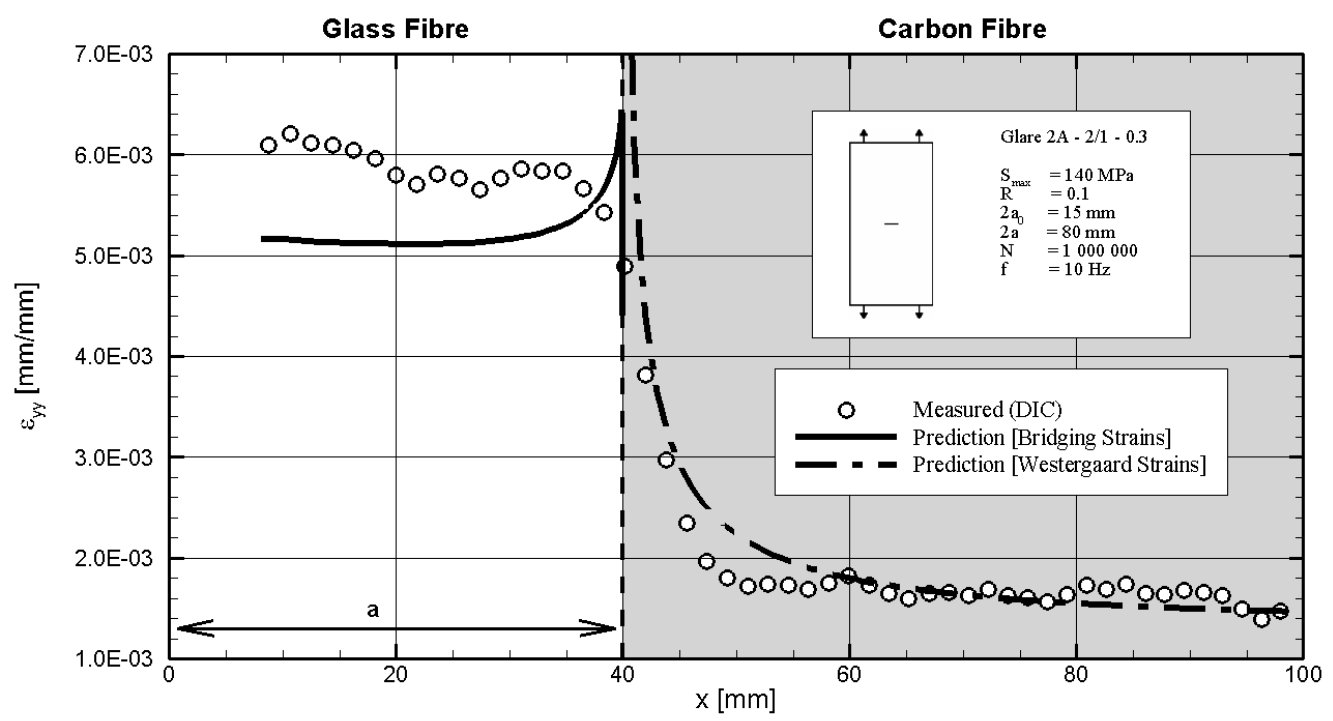

Figure 5.8: Comparison of predicted and measured fibre layer strains for CarbonGlass-Carbon configuration ITS-GLARE 2A-2/1-0.3 with $W=85 / 80 / 85 m m, \sigma_{\max }=$ $140 M P a, R=0.1,2 a_{0}=15 \mathrm{~mm}, N=1000000$. 
measured fibre layer strains, it can be stated that the load redistribution model is accurate. As such, the modifications made to the Alderliesten model are confirmed to work as intended and verification of the implemented model's fatigue crack growth and delamination shape predictions can continue.

\subsection{Verification}

Verification seeks to compare the model's predictions against real-world test data for which the model was developed. The implemented model's ability to accurately predict the fatigue behaviour of fibre metal laminates with local variations in fibre stiffness is carried out, with primary focus being on fatigue crack growth rates and secondary focus given to predicted delamination shapes.

\subsubsection{Crack growth rate verification}

The model's fatigue crack growth rate predictions are compared against three test cases: two specimens prepared for the purposes of this investigation and one from literature. The first two test cases consist of relatively simple GLARE 2A panels (this grade of GLARE contains only unidirectional fibres - no crossply fibre layers), under uniform tension. The third verification test for fatigue crack growth is carried out using data from previously published work by Rans [16] using a GLARE 3 panel.

Each of the panels were tested under uniaxial fatigue loading, with peak loads and stress ratios listed on the relevant figures. For the first two tests, visual measurements of the crack tip were made using a two-axis microscope stand and millimetre graph paper. This data was then run through a seven-point ASTM E647 floating curve fit to generate the crack growth rates (See Appendix C for the raw and processed 
data).

The first verification test consists of GLARE 2A-2/1-0.3 in a Carbon-Glass-Carbon configuration, the results for which are seen in Figure 5.9. The white area represents standard GLARE 2A while the shaded area represents the carbon fibre/epoxy substitution area. This test is used to verify the model's predictions for an increase in stiffness in the fibre layers. The model does not accurately predict the crack growth rate through the first portion of the panel (the glass section), however as the crack grows through the material transition, both the predicted and measured crack growth rates slow, and converge shortly afterwards. Once the crack tip has settled into the new material, the measured crack growth rate corresponds very well with the predicted crack growth rate.

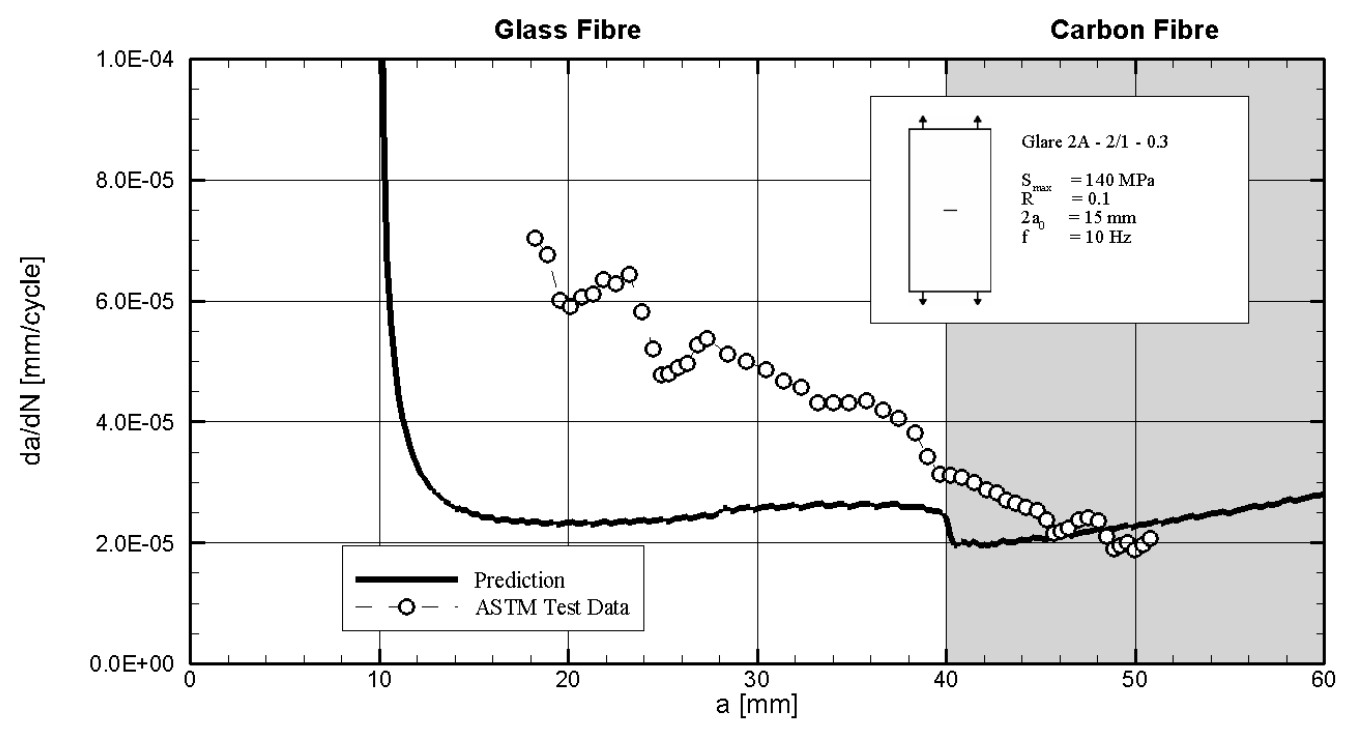

Figure 5.9: Comparison of predicted and measured crack growth rates for CarbonGlass-Carbon configuration ITS-GLARE 2A-2/1-0.3 with $W=85 / 80 / 85 m m, \sigma_{\max }=$ $140 M P a, R=0.1,2 a_{0}=15 \mathrm{~mm}$.

The second verification test consists of GLARE 2A-2/1-0.3 in a Carbon-Glass-Carbon configuration and is seen in Figure 5.10. Once again, the white area represents standard GLARE 2A while the shaded area represents the carbon fibre/epoxy substitution 
area. This test is used to verify the model's predictions for a decrease in stiffness in the fibre layers. From the initial growth in the carbon fiber section, the measured and predicted crack growth rates correspond reasonably well. Through the transition into the less stiff glass fibre section, the measured and predicted crack growth rates agree very well.

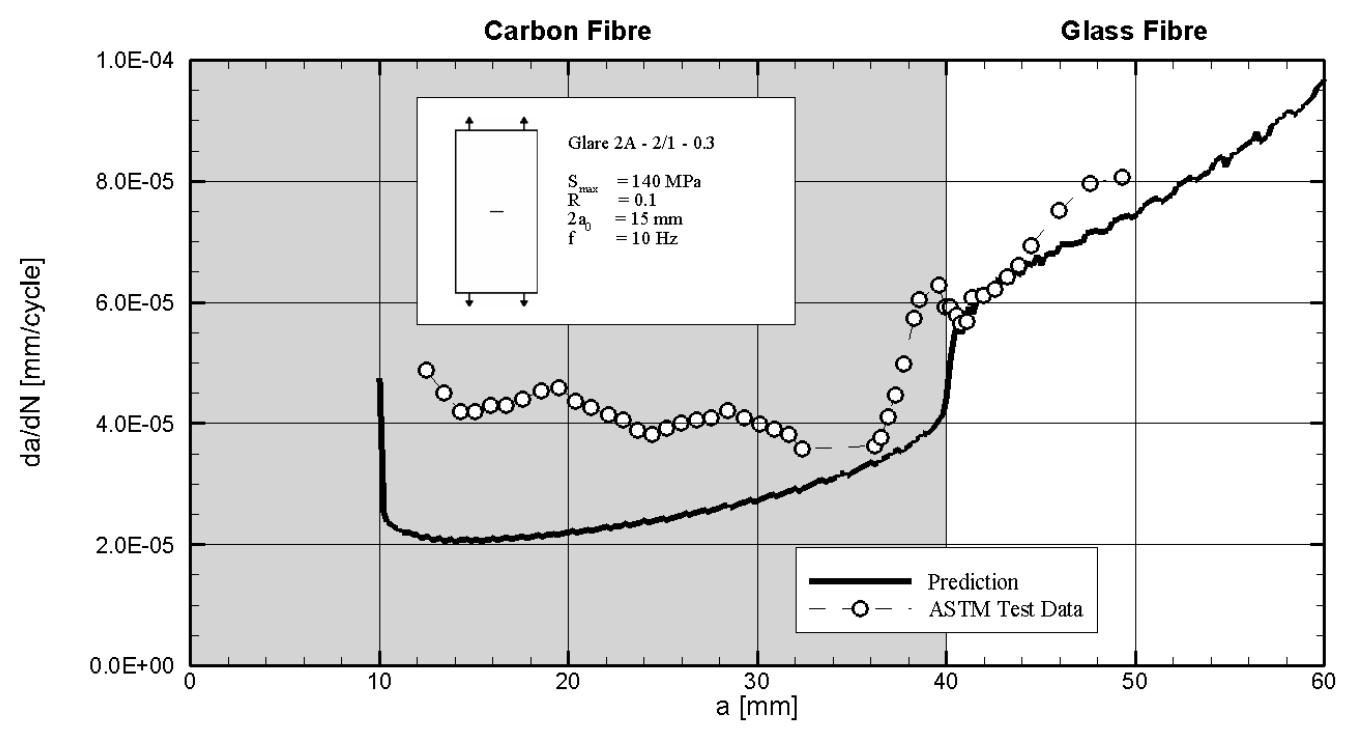

Figure 5.10: Comparison of predicted and measured crack growth rates for GlassCarbon-Glass configuration ITS-GLARE 2A-2/1-0.3 with $W=85 / 80 / 85 m m, \sigma_{\max }=$ $140 \mathrm{MPa}, R=0.1,2 a_{0}=15 \mathrm{~mm}$.

The final verification test was chosen from previously published work by Rans [16]. This test case was selected to expand the depth of the verification procedure, as the first two verification tests were limited to GLARE 2A, which does not contain crossply layers. As well, this selected test has two distinct stiffness transitions from the panel centre to the edge - more readily mimicking an internal stiffener. As with the previous two figures, the white area represents standard GLARE 2A while the shaded area represents the carbon fibre/epoxy substitution area.

As can be seen in Figure 5.11, there is good correspondence between the test data and the model predictions up to the first material transition. As the crack grows through 


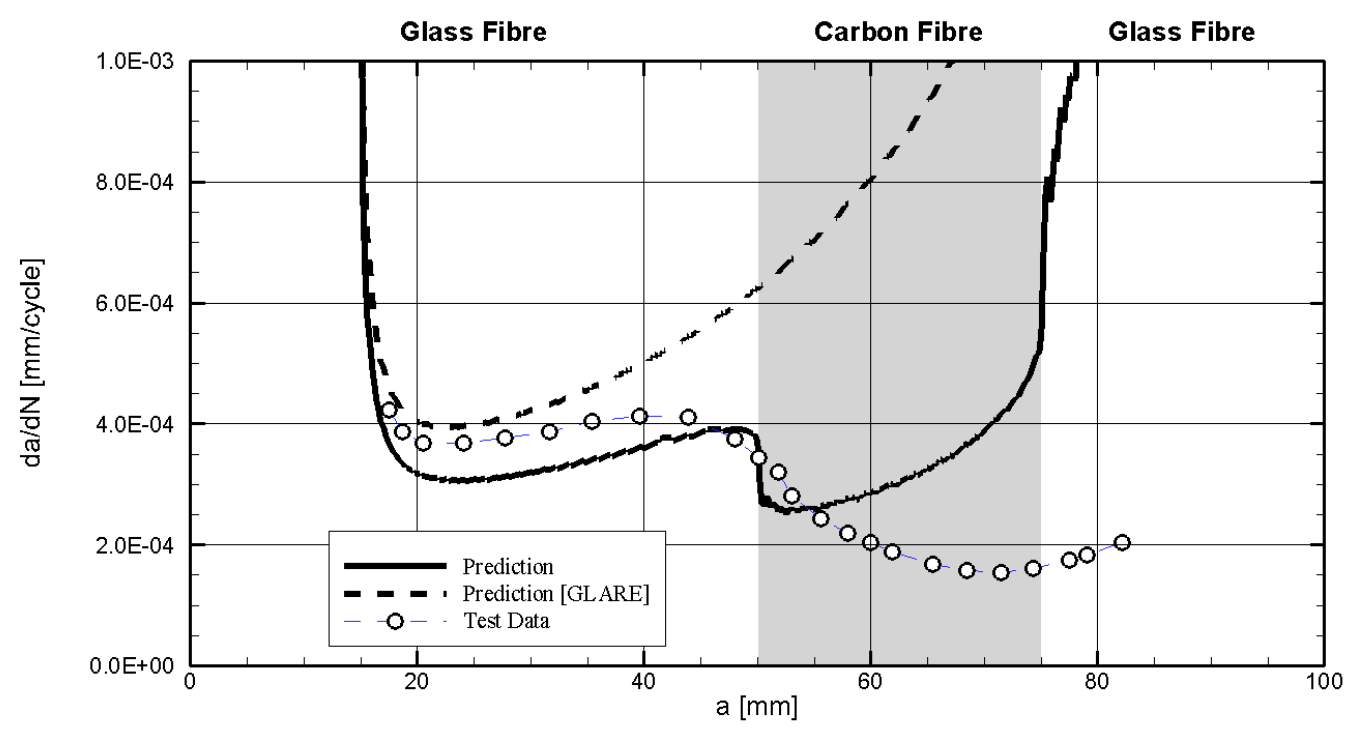

Figure 5.11: Crack growth rate comparison from literature [16] for GlassCarbon-Glass-Carbon-Glass configuration ITS-GLARE 3-3/2-0.4 with $W=$ $50 / 25 / 100 / 25 / 50 \mathrm{~mm}, \sigma_{\max }=120 M P a, R=0.05,2 a_{0}=25 \mathrm{~mm}$.

the internal stiffener, the model prediction and test results begin to diverge. This reflects the behaviour seen in the second test specimen - Figure 5.10 - an increase in crack growth rate as the crack tip approaches the lower stiffness fibre region. The test results show similar, yet reduced increase as the crack tip approaches the lower stiffness fibre region.

Once the crack enters the lower stiffness region after the internal stiffener, the predicted crack growth rate increases quite rapidly while the measured crack growth rate remains retarded. The predicted behaviour mimics that seen in Figure 5.10 - a ramp up of the crack growth rates is present as the crack tip approaches a stiffness reduction. The final predicted crack growth rate outside of the simulated stiffener $(a>75 \mathrm{~mm})$ is still less than that of a standard GLARE sheet. This agrees with conclusion of Rans [16] that the internal stiffener continues to retard the crack growth rate once the crack has grown through this section, mainly due to higher bridging stiffness. 


\subsubsection{Delamination Shape Verification}

The final step in the validation and verification process is to verify the model's ability to accurately predict the delamination shape, both overall and close to the crack tip. Again, as mentioned earlier in Section 5.3.3, the delamination zones closer to the crack tip have a larger impact on the bridging solution, and as a result accuracy here is key. Once again, two test cases are presented - one with the crack growing through a drop in stiffness (Figure 5.13 and another with the crack growing through an increase in stiffness (Figure 5.12. The panel configuration are the same as those shown in Figures 5.9 and 5.10, with the panels etched in a nitric acid solution after the final crack growth measurement were taken.

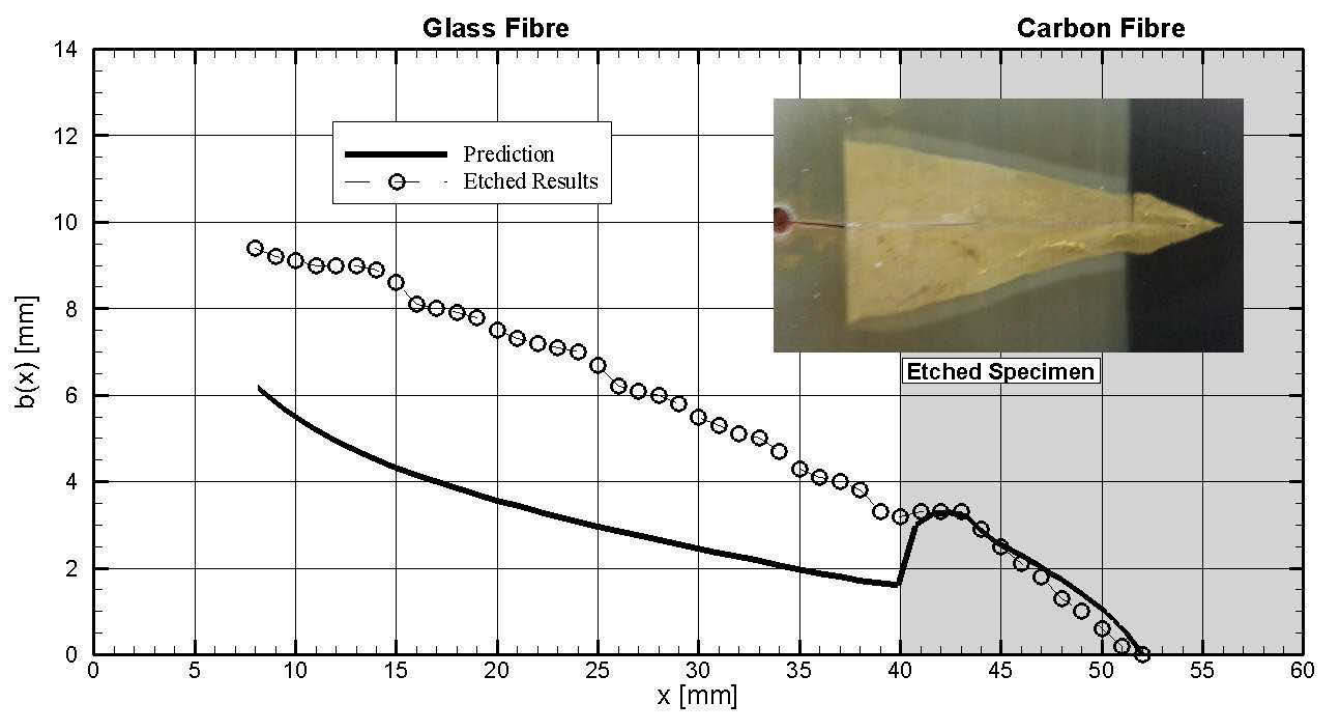

Figure 5.12: Comparison of predicted and measured delamination sizes for CarbonGlass-Carbon configuration ITS-GLARE 2A-2/1-0.3 with $W=85 / 80 / 85 m m, \sigma_{\max }=$ $140 M P a, R=0.1,2 a_{0}=15 m m, 2 a_{f}=104 m m, N_{f}=1000000$.

The first delamination comparison uses a GLARE 2A-2/1-0.3 panel with CarbonGlass-Carbon configuration. Similar to the validation comparisons done with the Alderliesten data/model, the predicted and measured delamination shapes correspond quite well at the crack tip and begin to deviate further from the crack tip. 
Larger measured delaminations are seen, with the model underpredicting the shape close to the sawcut edge. Clearly the cumulative error that results from the model implementation has an even greater effect here.

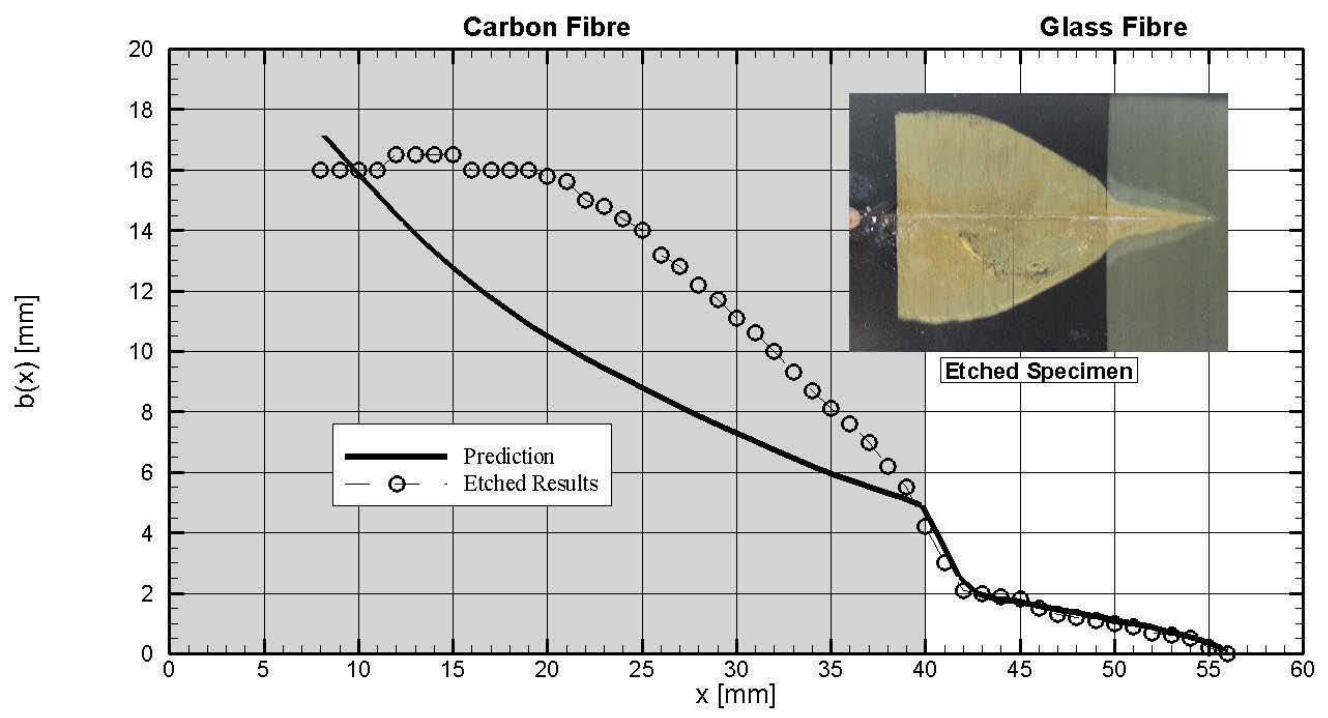

Figure 5.13: Comparison of predicted and measured delamination sizes for GlassCarbon-Glass configuration ITS-GLARE 2A-2/1-0.3 with $W=85 / 80 / 85 m m, \sigma_{\max }=$ $140 \mathrm{MPa}, R=0.1,2 a_{0}=15 \mathrm{~mm}, 2 a_{f}=112 \mathrm{~mm}, N_{f}=1000000$.

The second delamination comparison uses a GLARE 2A-2/1-0.3 panel with GlassCarbon-Glass configuration. The measured delamination shape agrees very well with the predicted delamination shape, with slight differences noted in the region between the sawcut and the material transition - though this difference is relatively small. The predicted and measured delamination shape in the region surrounding the crack tip correspond very well.

Overall, the model succeeds in accurately predicting the delamination shape close to the crack tip in both verification and validation test cases. As it is this region that has the greatest influence on the bridging stress solution, it can be concluded that the model is performing the task of delamination shape prediction sufficiently well. 


\subsection{Summary}

The new model maintains fidelity with the Alderliesten model predictions of crack opening displacement, crack growth rates and delamination shapes. Some deviation is seen in the crack growth rate predictions for large crack-to-width ratios. The implementation of the load redistribution model is successfully validated, with little deviation present between the measured and predicted fibre layer strains.

The implemented model shows relatively good correlation between the predicted and measured fatigue crack growth rates (excellent correspondence for two of the three test cases, reasonably good correspondence for the third test case). Small deviations in bridging stress result in similar deviations in the bridging stress intensity factor and the final effective stress intensity factor which lead to small initial differences in fatigue crack growth rates. This difference compounds with larger crack-to-width ratios. Despite this, the deviation in crack growth rate predictions are within the same order of magnitude as the measured growth rates. This result would allow the model to be used with relatively conservative fatigue knockdown factors.

For delamination shape predictions, the model accurately predicts the shape of the delamination zones closest to the crack tip, with reducing accuracy further away from the crack tip. This is consistent with the Alderliesten model behaviour, and again is as a result of cumulative error over the calculation loops. Further study of the delamination behaviour of 2024-T3 Al and the materials involved should reduce this uncertainty and minimize the error involved.

Finally, one must consider what is and what is not the model resulting from the present study when examining the overall agreement between test and prediction in this chapter. At its core, the modifications made to the Alderliesten FML crack growth model in this thesis reflect a redistribution of load based on local variations 
in the fibre layer both ahead and behind the crack tip. The most appropriate comparisons in terms of the impact of the modifications proposed in this thesis are thus measurements of the fibre strain distributions (Section 5.4) and crack opening displacements (Section 5.3.1. Ultimately, it is the damage growth rate, and more importantly damage size (both crack and delamination) that are of primary concern from a design standpoint. However, in comparing the the results for predictions and tests for this measure, one must keep in mind several key aspects.

First, damage growth rates (both crack and delamination) are both determined based on the application of empirical Paris relation models (Section 4.2.1). These models are sensitive to their empirical material property inputs and loading. The power-law nature of the empirical material behaviour amplifies the sensitivity of the predicted damage growth rate to small changes in loading and small inaccuracies in material characterization. In the present study, the loading and load redistribution aspects of the model were found to be in good agreement (Section 5.4). Thus, the deviations between predicted and observed damage growth behaviour are expected to be a result of inaccuracies in the material behaviour inputs used in this study (which were obtained from literature) and/or a high sensitivity to the accuracy of the load redistribution predictions.

Second, damage size is determined based upon an integration of damage growth rates over the number of applied fatigue cycles. As such, any errors observed in predicting growth rates will accumulate over the number of fatigue cycles. This is most easily visualized in the delamination size predictions given in Figure 5.5 where prediction of delamination size near the crack tip is more accurate as it is a result of less fatigue cycles, while away from the crack tip where the delamination has been exposed to more fatigue cycles, the deviation between prediction and observation increases.

These issues with prediction of damage growth rates and damage size are a reality for 
design engineers. Based on the validation and verification work presented here, one could argue that the developed model accurately captures the intended behaviour - the load redistribution within the FML due to local variations in fibre stiffness, yet manifests significant errors in terms of the desired output - damage size. This warrants the conclusion that the developed model achieves its scientific aim, yet requires caution and thorough sensitivity analysis when applied in practice for the prediction of the size and growth rate of fatigue damage. 


\section{Chapter 6}

\section{Conclusions and Future Work}

\subsection{Conclusions}

The investigation presented in this thesis set out to develop, implement and validate a simple analytical model for crack propagation within fibre metal laminates with local variations in fibre stiffness. As use of fibre metal laminates increase in aerospace applications, structurally optimized laminates are becoming more desirable. This work was motivated by an initial investigation into the crack arresting capacity of internal carbon fibre tear straps in GLARE. This investigation sought to evaluate and model the effect of two main cases on fatigue crack growth in fibre metal laminates:

1. A variation in stiffness in the fibre layers ahead of the crack tip

2. A variation in stiffness in the fibre layers behind the crack tip

The investigation modified the Alderliesten fatigue crack growth model to accommodate for local variations in fibre layer stiffness using a load redistribution model. Testing was carried out to verify the model's fatigue crack growth predictions as well as to validate the load redistribution model. 
Experimental validation and verification of the implemented model showed:

- good correlation between measured and predicted strains in the fibre and metal layers

- good correlation between measured and predicted fatigue crack growth rates

- accurate prediction of the delamination shape close to the crack tip.

The modified model deviated from validation data for large crack-to-width ratios, the result of differing finite width correction factors. The load redistribution model was found to be accurate, however the implemented model was found to be sensitive to small errors cumulating over repeated cycles. This cumulative error results in larger deviations between prediction and measured crack growth rates for larger crack sizes.

As it is the crack growth and delamination propagation rates that are critical to damage tolerant design, this sensitivity must be taken into account when using this tool to optimize structures for fatigue loading.

\subsection{Future Work}

This refined fatigue crack growth prediction model has great potential for future applications in damage tolerant design. Several investigations to further this work are proposed below.

Since the model was shown to be sensitive to the empirical Paris relation constants, more rigorous study of the model's sensitivity to these inputs should be carried out before any designs are optimized using this tool. In particular, proper statistical analysis of the Paris relation constants and their relative sensitivities should allow for an accurate knockdown factor to be calculated for this model. 
Further investigation of the effect of large crack-to-width ratios on fatigue crack growth and crack bridging should be carried out. While practical designs should not experience such large crack-to-width ratios, better understanding of the mechanisms driving this difference should lead to a more complete understanding of the overall fatigue crack growth process.

Now that the crack arresting behaviour of 'internal tear straps' has been characterized and can be accurately predicted, further research should focus on the feasibility of these crack arrest features in aerospace structures. To achieve this, further work should extend this model to study biaxial fatigue loading and the possibility of crack bifurcation within such a structure. 


\section{List of References}

[1] C. Rans. An experimental investigation into the fatigue behaviour of dimple countersunk GLARE riveted lap joints. Ph.D. thesis, Carleton University (2004).

[2] A. Vlot and J. W. Gunnink. Fibre Metal Laminates an introduction. Kluwer Academic Publishers, Dordrecht, The Netherlands (2001).

[3] C. Pitzer and J.-M. Yang. "Hybrid Metal Laminate Manufacturing Planning Evaluation and Assessment." Technical report, Department of Materials Science and Engineering - University of California, Los Angeles (2008).

[4] G. Wilson. Fatigue Crack Growth Prediction for generalized fiber metal laminates and hybrid materials. Ph.D. thesis, Delft University of Technology (2013).

[5] H. A. Wood, R. M. Engle, R. L. Kuster, and D. L. Smith. "USAF damage tolerant design handbook: guidelines for the analysis and design of damage tolerant aircraft." Technical report, Air Force Flight Dynamics Laboratory, WrightPatterson Air Force Base, Ohio (1979).

[6] R. Alderliesten and A. Vlot. "Modelling fatigue crack growth characteristics of GLARE." In "ICCM-13," (2001).

[7] R. Alderliesten. Fatigue crack propagation and delamination growth in Glare. Ph.D. thesis, Delft University of Technology (2005).

[8] R. Alderliesten. "On the available relevant approaches for fatigue crack propagation prediction in Glare." International Journal of Fatigue 29(2), 289-304. ISSN 01421123 (2007).

[9] T. Takamatsu, T. Shimokawa, T. Matsumura, Y. Miyoshi, and Y. Tanabe. "Evaluation of fatigue crack growth behavior of GLARE3 fiber/metal laminates using a compliance method." Engineering Fracture Mechanics 70(18), 2603-2616. ISSN 00137944 (2003). 
[10] Y. Guo and X. Wu. "A phenomenological model for predicting crack growth in fiber-reinforced metal laminates under constant-amplitude loading." Composites science and technology 59 (1999).

[11] B. Cox. "Life Prediction for Bridged Fatigue Cracks." In W. Johnson, J. Larsen, and B. Cox, editors, "Life Prediction for Titanium Matrix Composites," pages 552-572. ASTM. ISBN 052143064X (1996).

[12] B. Bolker. Ecological Models and Data in R. Princeton University Press. ISBN 9781400840908 (2008).

[13] R. Marissen. "Fatigue crack growth in ARALL, A hybrid aluminium-aramid composite material: Crack growth mechanisms and quantitative predictions of the crack growth rates." (1988).

[14] Y. Guo and X. Wu. "A Theoretical Model for Predicting Fatigue Crack Growth Rates in Fibre-Reinforced Metal Laminates." Fatigue $\mathcal{E}_{3}$ Fracture of Engineering Materials and Structures 21(9), 1133-1145. ISSN 8756-758X (1998).

[15] R. Alderliesten. "Analytical prediction model for fatigue crack propagation and delamination growth in Glare." International Journal of Fatigue 29(4), 628-646. ISSN 01421123 (2007).

[16] C. Rans, F. Morinière, R. Rodi, R. Alderliesten, and R. Benedictus. "Fatigue Behavior of Fiber/Metal Laminate Panels Containing Internal Carbon Tear Straps." Journal of Aircraft 48(6), 2122-2129. ISSN 0021-8669 (2011).

[17] J. Schijve. "Some formulas for the crack opening stress level." Engineering Fracture Mechanics pages 461-465 (1981).

[18] H. Westergaard. "Bearing pressures and cracks." Journal of Applied Mechanics 61, A49-53 (1939).

[19] Department of Defense. "DoD Modeling and Simulation (M\&S) Verification, Validation, and Accreditation." (2009).

[20] R. Rodi, R. Alderliesten, and R. Benedictus. "An experimental approach to investigate detailed failure mechanisms in Fibre Metal Laminates." ICAF 2009, Bridging the Gap Between Theory and Operational Practice (May), 27-29 (2009). 


\section{Appendix A}

\section{Material Properties}

The following material properties have been taken from literature and manufacturer's data, with the exception of the delamination constants for Mode II delamination growth in the carbon fibre (MD30SC with DT120 prepreg). These constants were determined from previous work done at Carleton University on Mode II delamination growth in a carbon-carbon interface (See Appendix E for this calculation.

Table A.1: Properties of GLARE and stiffener constituent materials

\begin{tabular}{cccc}
\hline Property [units] & 2024-T3 aluminium & UD S2 glass/FM94 & UD M30SC/DT120 \\
\hline \hline$E_{11}[\mathrm{MPa}]$ & 72400 & 48900 & 145000 \\
$E_{22}[\mathrm{MPa}]$ & 72400 & 5500 & 7800 \\
$G_{12}[\mathrm{MPa}]$ & 27600 & 5500 & 5500 \\
$\nu_{12}$ & 0.33 & 0.33 & 0.27 \\
$\nu_{13}$ & 0.33 & 0.0371 & 0.022 \\
$\alpha_{0^{\circ}}\left[\frac{1}{{ }^{\circ} \mathrm{C}}\right]$ & $2.2 \times 10^{-5}$ & $6.1 \times 10^{-5}$ & $-4.5 \times 10^{-5}$ \\
$\alpha_{90^{\circ}}\left[\frac{1}{{ }^{\circ} \mathrm{C}}\right]$ & $2.2 \times 10^{-5}$ & $2.6 \times 10^{-5}$ & $2.6 \times 10^{-5}$ \\
$\mathrm{C}$ & $3.689 \times 10^{-13}$ & 0.05 & 0.0943 \\
$\mathrm{~m}$ & 3.591 & 7.5 & 5.8648 \\
\hline
\end{tabular}




\section{Appendix B}

\section{Test Specimens}

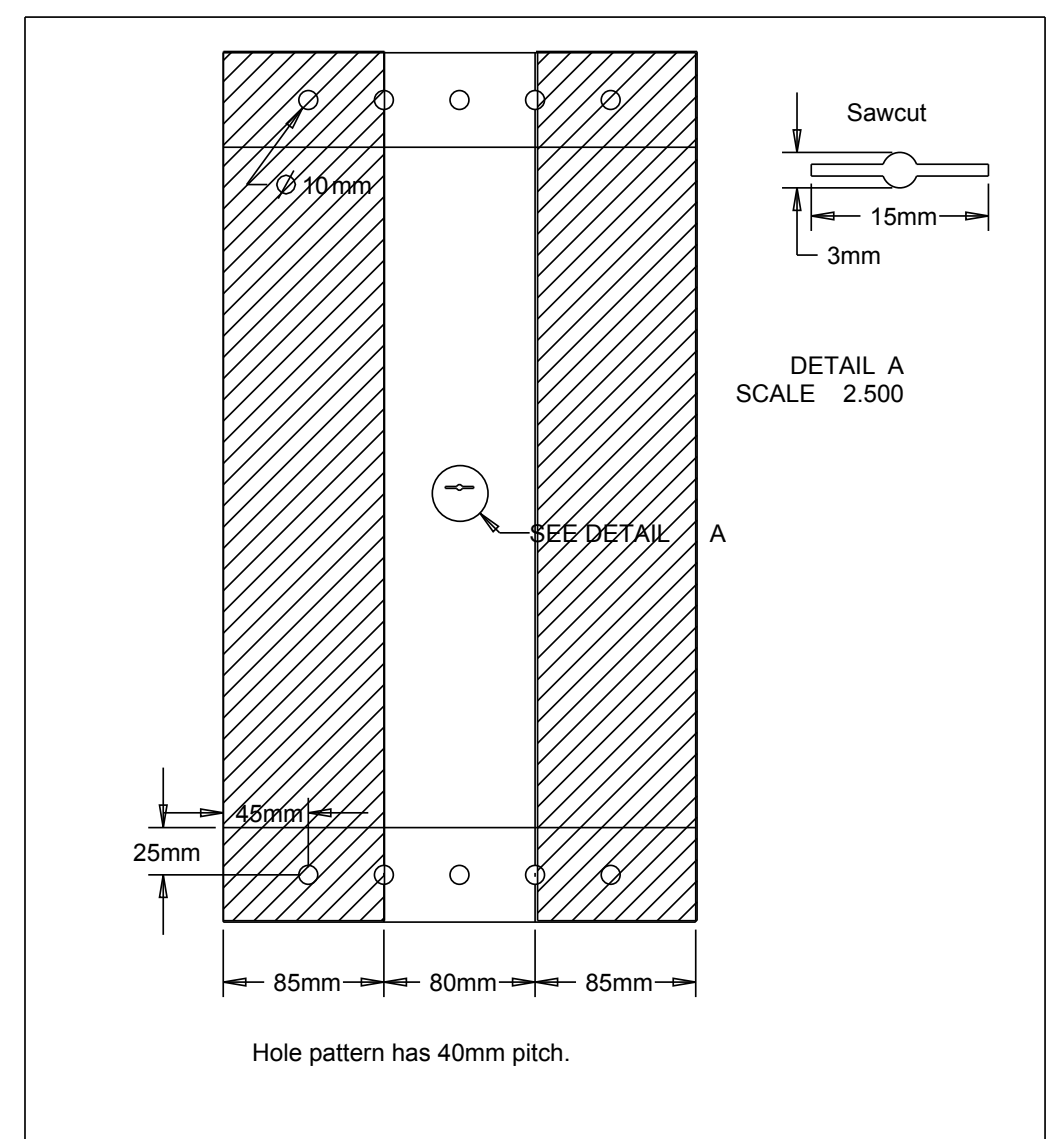

Figure B.1: Carbon-Glass-Carbon Fatigue Test Specimen Configuration 


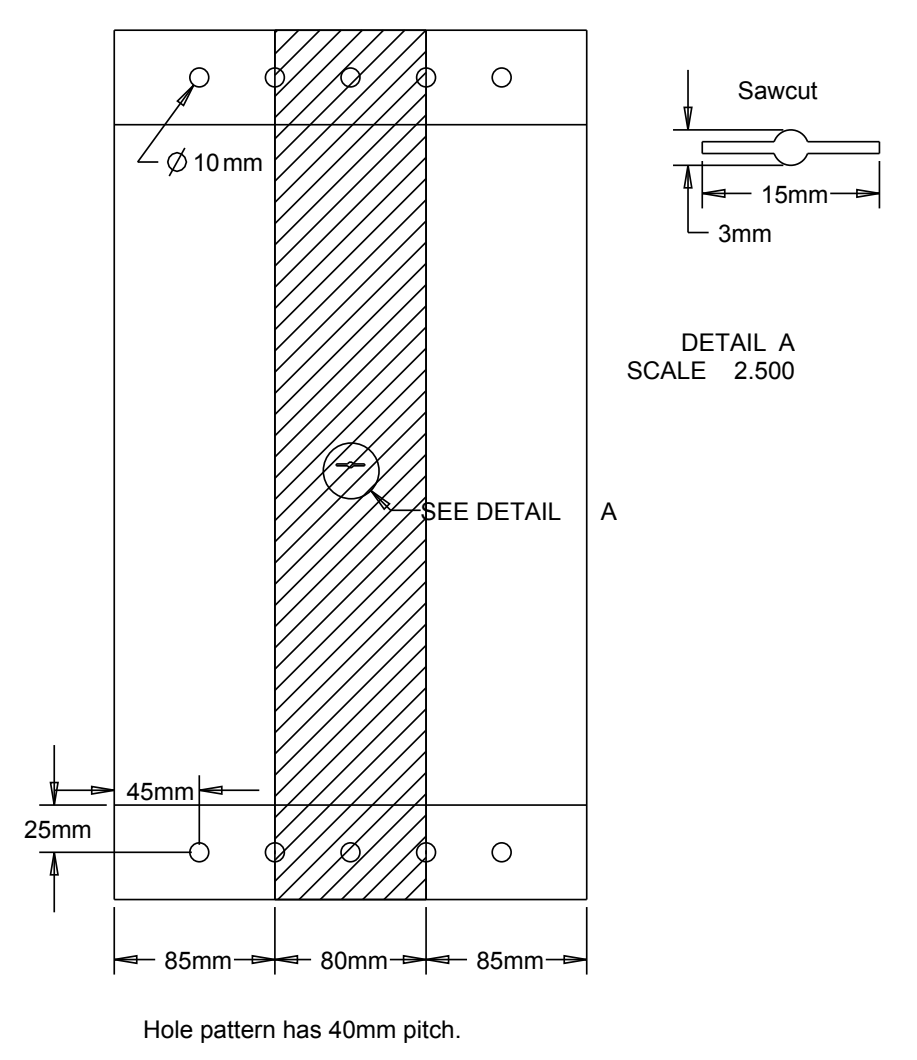

Figure B.2: Glass-Carbon-Glass Fatigue Test Specimen Conffguration 


\section{Appendix $\mathrm{C}$}

\section{Crack Growth Data}

Table C.1: ASTM Crack Growth Data for Carbon-Glass-

Carbon Panel, $W=85 / 80 / 85, \sigma_{\max }=140 M P a, R=0.10$, $2 a_{0}=15 \mathrm{~mm}$

\begin{tabular}{|l|l|l|l|l|l|l|}
\hline CES No. & CYCLES & A(meas.) & A(reg.) & M.C.C. & Delt. K & DA/DN \\
\hline \hline 1 & 10000 & 15.85 & & & & \\
\hline 2 & 20000 & 16.58 & & & & \\
\hline 3 & 30000 & 17.57 & & & & \\
\hline 4 & 40000 & 18.39 & 18.24429 & 0.995699 & 966.645 & $7.04 \mathrm{E}-05$ \\
\hline 5 & 50000 & 18.79 & 18.89286 & 0.987907 & 984.6299 & $6.76 \mathrm{E}-05$ \\
\hline 6 & 60000 & 19.46 & 19.51714 & 0.977357 & 1001.732 & $6.01 \mathrm{E}-05$ \\
\hline 7 & 70000 & 20.09 & 20.07667 & 0.968822 & 1016.898 & $5.91 \mathrm{E}-05$ \\
\hline 8 & 80000 & 20.85 & 20.69952 & 0.964485 & 1033.61 & $6.06 \mathrm{E}-05$ \\
\hline 9 & 90000 & 21.11 & 21.26762 & 0.958846 & 1048.708 & $6.11 \mathrm{E}-05$ \\
\hline
\end{tabular}




\begin{tabular}{|c|c|c|c|c|c|c|}
\hline CES No. & CYCLES & A(meas.) & A(reg.) & M.C.C. & Delt. K & $\mathrm{DA} / \mathrm{DN}$ \\
\hline 10 & 100000 & 21.9 & 21.85286 & 0.957112 & 1064.125 & $6.35 \mathrm{E}-05$ \\
\hline 11 & 110000 & 22.48 & 22.52048 & 0.946974 & 1081.556 & $6.28 \mathrm{E}-05$ \\
\hline 12 & 120000 & 23.22 & 23.23429 & 0.946548 & 1100.021 & $6.43 \mathrm{E}-05$ \\
\hline 13 & 130000 & 23.98 & 23.87095 & 0.9317 & 1116.351 & $5.83 \mathrm{E}-05$ \\
\hline 14 & 140000 & 24.36 & 24.45524 & 0.913138 & 1131.231 & $5.21 \mathrm{E}-05$ \\
\hline 15 & 150000 & 24.97 & 24.88667 & 0.885883 & 1142.158 & $4.77 \mathrm{E}-05$ \\
\hline 16 & 160000 & 25.3 & 25.26952 & 0.878519 & 1151.812 & $4.8 \mathrm{E}-05$ \\
\hline 17 & 170000 & 25.63 & 25.77762 & 0.87184 & 1164.569 & $4.89 \mathrm{E}-05$ \\
\hline 18 & 180000 & 26.26 & 26.26942 & 0.896999 & 1176.858 & $4.96 \mathrm{E}-05$ \\
\hline 19 & 190000 & 26.97 & 26.8349 & 0.92236 & 1190.924 & $5.27 \mathrm{E}-05$ \\
\hline 20 & 200000 & 27.27 & 27.34351 & 0.941862 & 1203.518 & $5.38 \mathrm{E}-05$ \\
\hline 21 & 220000 & 28.44 & 28.4051 & 0.948405 & 1229.652 & $5.12 \mathrm{E}-05$ \\
\hline 22 & 240000 & 29.32 & 29.39319 & 0.954086 & 1253.81 & $5 \mathrm{E}-05$ \\
\hline 23 & 260000 & 30.52 & 30.44476 & 0.954816 & 1279.374 & 4.87E-05 \\
\hline 24 & 280000 & 31.32 & 31.3781 & 0.948707 & 1301.957 & $4.67 \mathrm{E}-05$ \\
\hline 25 & 300000 & 32.4 & 32.29905 & 0.944108 & 1324.162 & $4.57 \mathrm{E}-05$ \\
\hline 26 & 320000 & 33.06 & 33.16571 & 0.935569 & 1345.001 & $4.32 \mathrm{E}-05$ \\
\hline 27 & 340000 & 34.04 & 34.01857 & 0.933666 & 1365.467 & $4.32 \mathrm{E}-05$ \\
\hline 28 & 360000 & 34.92 & 34.83476 & 0.932336 & 1385.027 & $4.32 \mathrm{E}-05$ \\
\hline
\end{tabular}




\begin{tabular}{|c|c|c|c|c|c|c|}
\hline CES No. & CYCLES & A(meas.) & A(reg.) & M.C.C. & Delt. K & $\mathrm{DA} / \mathrm{DN}$ \\
\hline 29 & 380000 & 35.63 & 35.75048 & 0.931784 & 1406.953 & $4.35 \mathrm{E}-05$ \\
\hline 30 & 400000 & 36.61 & 36.61905 & 0.924372 & 1427.746 & $4.19 \mathrm{E}-05$ \\
\hline 31 & 420000 & 37.57 & 37.46762 & 0.917876 & 1448.067 & $4.06 \mathrm{E}-05$ \\
\hline 32 & 440000 & 38.27 & 38.31048 & 0.906455 & 1468.269 & $3.81 \mathrm{E}-05$ \\
\hline 33 & 460000 & 38.98 & 39.01571 & 0.883559 & 1485.193 & $3.43 \mathrm{E}-05$ \\
\hline 34 & 480000 & 39.72 & 39.63857 & 0.859677 & 1500.161 & $3.14 \mathrm{E}-05$ \\
\hline 35 & 500000 & 40.2 & 40.2181 & 0.853528 & 1514.109 & $3.12 \mathrm{E}-05$ \\
\hline 36 & 520000 & 40.78 & 40.8081 & 0.848786 & 1528.333 & $3.09 \mathrm{E}-05$ \\
\hline 37 & 540000 & 41.36 & 41.43857 & 0.833647 & 1543.565 & $2.99 \mathrm{E}-05$ \\
\hline 38 & 560000 & 42.15 & 42.09714 & 0.816708 & 1559.515 & $2.88 \mathrm{E}-05$ \\
\hline 39 & 580000 & 42.75 & 42.64952 & 0.801603 & 1572.927 & $2.82 \mathrm{E}-05$ \\
\hline 40 & 600000 & 43.15 & 43.18 & 0.774974 & 1585.839 & $2.7 \mathrm{E}-05$ \\
\hline 41 & 620000 & 43.53 & 43.64048 & 0.764161 & 1597.074 & $2.66 \mathrm{E}-05$ \\
\hline 42 & 640000 & 44.26 & 44.20667 & 0.737602 & 1610.926 & $2.58 \mathrm{E}-05$ \\
\hline 43 & 660000 & 44.73 & 44.77714 & 0.71907 & 1624.928 & $2.53 \mathrm{E}-05$ \\
\hline 44 & 680000 & 45.43 & 45.29714 & 0.679143 & 1637.731 & $2.38 \mathrm{E}-05$ \\
\hline 45 & 700000 & 45.64 & 45.69857 & 0.59936 & 1647.644 & $2.16 \mathrm{E}-05$ \\
\hline 46 & 720000 & 46.07 & 46.07476 & 0.60305 & 1656.957 & $2.2 \mathrm{E}-05$ \\
\hline 47 & 740000 & 46.46 & 46.47 & 0.618625 & 1666.767 & $2.25 \mathrm{E}-05$ \\
\hline
\end{tabular}




\begin{tabular}{|c|c|c|c|c|c|c|}
\hline CES No. & CYCLES & A(meas.) & A(reg.) & M.C.C. & Delt. K & $\mathrm{DA} / \mathrm{DN}$ \\
\hline 48 & 760000 & 46.93 & 47.00095 & 0.65037 & 1679.989 & $2.38 \mathrm{E}-05$ \\
\hline 49 & 780000 & 47.57 & 47.5 & 0.656025 & 1692.463 & $2.42 \mathrm{E}-05$ \\
\hline 50 & 800000 & 48.06 & 48.01381 & 0.636348 & 1705.355 & $2.36 \mathrm{E}-05$ \\
\hline 51 & 820000 & 48.39 & 48.49333 & 0.545032 & 1717.435 & $2.1 \mathrm{E}-05$ \\
\hline 52 & 840000 & 48.92 & 48.85143 & 0.432485 & 1726.487 & $1.9 \mathrm{E}-05$ \\
\hline 53 & 860000 & 49.26 & 49.17619 & 0.453808 & 1734.72 & $1.96 \mathrm{E}-05$ \\
\hline 54 & 880000 & 49.44 & 49.5719 & 0.461432 & 1744.782 & $2 \mathrm{E}-05$ \\
\hline 55 & 900000 & 49.91 & 49.9781 & 0.381892 & 1755.148 & $1.89 \mathrm{E}-05$ \\
\hline 56 & 920000 & 50.53 & 50.37952 & 0.407739 & 1765.431 & $1.96 \mathrm{E}-05$ \\
\hline 57 & 940000 & 50.83 & 50.7919 & 0.455716 & 1776.034 & $2.08 \mathrm{E}-05$ \\
\hline 58 & 960000 & 51.04 & & & & \\
\hline 59 & 980000 & 51.55 & & & & \\
\hline 60 & 1000000 & 52.05 & & & & \\
\hline
\end{tabular}

Table C.2: ASTM Crack Growth Data for Glass-Carbon-

Glass Panel, $W=85 / 80 / 85, \sigma_{\max }=140 M P a, R=0.10$,

$2 a_{0}=15 \mathrm{~mm}$

\begin{tabular}{|l|l|l|l|l|l|l|}
\hline CES No. & CYCLES & A(meas.) & A(reg.) & M.C.C. & Delt. K & DA/DN \\
\hline \hline 1 & 20000 & 9.22 & & & & \\
\hline
\end{tabular}




\begin{tabular}{|c|c|c|c|c|c|c|}
\hline CES No. & CYCLES & A(meas.) & A(reg.) & M.C.C. & Delt. K & $\mathrm{DA} / \mathrm{DN}$ \\
\hline 2 & 40000 & 10.44 & & & & \\
\hline 3 & 60000 & 11.55 & & & & \\
\hline 4 & 80000 & 12.46 & 12.46762 & 0.999069 & 793.4395 & $4.88 \mathrm{E}-05$ \\
\hline 5 & 100000 & 13.31 & 13.39857 & 0.998026 & 823.3185 & $4.5 \mathrm{E}-05$ \\
\hline 6 & 120000 & 14.3 & 14.27143 & 0.996653 & 850.532 & $4.19 \mathrm{E}-05$ \\
\hline 7 & 140000 & 15.17 & 15.05381 & 0.992235 & 874.3353 & 0.000042 \\
\hline 8 & 160000 & 15.82 & 15.84857 & 0.987732 & 898 & $4.29 \mathrm{E}-05$ \\
\hline 9 & 180000 & 16.51 & 16.68714 & 0.984276 & 922.4582 & $4.3 \mathrm{E}-05$ \\
\hline 10 & 200000 & 17.66 & 17.58238 & 0.980885 & 948.0448 & $4.4 \mathrm{E}-05$ \\
\hline 11 & 220000 & 18.63 & 18.53286 & 0.979842 & 974.6755 & $4.54 \mathrm{E}-05$ \\
\hline 12 & 240000 & 19.41 & 19.45857 & 0.978597 & 1000.136 & $4.59 \mathrm{E}-05$ \\
\hline 13 & 260000 & 20.29 & 20.33429 & 0.975395 & 1023.831 & $4.37 \mathrm{E}-05$ \\
\hline 14 & 280000 & 21.2 & 21.20619 & 0.973054 & 1047.082 & $4.26 \mathrm{E}-05$ \\
\hline 15 & 300000 & 22.16 & 22.08714 & 0.970986 & 1070.261 & $4.14 \mathrm{E}-05$ \\
\hline 16 & 320000 & 22.86 & 22.87714 & 0.968091 & 1090.804 & $4.06 \mathrm{E}-05$ \\
\hline 17 & 340000 & 23.66 & 23.66571 & 0.963371 & 1111.1 & $3.89 \mathrm{E}-05$ \\
\hline 18 & 360000 & 24.34 & 24.40286 & 0.960666 & 1129.901 & $3.82 \mathrm{E}-05$ \\
\hline 19 & 380000 & 25.28 & 25.16619 & 0.961299 & 1149.21 & $3.92 \mathrm{E}-05$ \\
\hline 20 & 400000 & 25.88 & 25.94667 & 0.961427 & 1168.8 & $4 \mathrm{E}-05$ \\
\hline
\end{tabular}




\begin{tabular}{|c|c|c|c|c|c|c|}
\hline CES No. & CYCLES & A(meas.) & A(reg.) & M.C.C. & Delt. K & $\mathrm{DA} / \mathrm{DN}$ \\
\hline 21 & 420000 & 26.73 & 26.77238 & 0.960865 & 1189.372 & $4.05 \mathrm{E}-05$ \\
\hline 22 & 440000 & 27.62 & 27.5619 & 0.960737 & 1208.911 & $4.1 \mathrm{E}-05$ \\
\hline 23 & 460000 & 28.45 & 28.43095 & 0.96242 & 1230.285 & $4.22 \mathrm{E}-05$ \\
\hline 24 & 480000 & 29.2 & 29.28905 & 0.959186 & 1251.271 & $4.1 \mathrm{E}-05$ \\
\hline 25 & 500000 & 30.15 & 30.09762 & 0.955929 & 1270.95 & $3.99 \mathrm{E}-05$ \\
\hline 26 & 520000 & 30.95 & 30.88227 & 0.978977 & 1289.971 & $3.9 \mathrm{E}-05$ \\
\hline 27 & 540000 & 31.59 & 31.64663 & 0.982995 & 1308.439 & $3.81 \mathrm{E}-05$ \\
\hline 28 & 560000 & 32.39 & 32.35105 & 0.983021 & 1325.414 & $3.58 \mathrm{E}-05$ \\
\hline 29 & 670000 & 36.1 & 36.18135 & 0.981044 & 1417.268 & $3.63 \mathrm{E}-05$ \\
\hline 30 & 680000 & 36.58 & 36.54099 & 0.975588 & 1425.877 & $3.76 \mathrm{E}-05$ \\
\hline 31 & 690000 & 36.88 & 36.91759 & 0.959509 & 1434.894 & $4.11 \mathrm{E}-05$ \\
\hline 32 & 700000 & 37.33 & 37.30608 & 0.810953 & 1444.198 & $4.47 \mathrm{E}-05$ \\
\hline 33 & 710000 & 37.7 & 37.74126 & 0.856466 & 1454.623 & $4.98 \mathrm{E}-05$ \\
\hline 34 & 720000 & 38.24 & 38.2747 & 0.877943 & 1467.411 & $5.73 \mathrm{E}-05$ \\
\hline 35 & 725000 & 38.62 & 38.56491 & 0.879046 & 1474.372 & $6.04 \mathrm{E}-05$ \\
\hline 36 & 740000 & 39.56 & 39.5823 & 0.857612 & 1498.808 & $6.28 \mathrm{E}-05$ \\
\hline 37 & 745000 & 39.95 & 39.92633 & 0.814304 & 1507.084 & $5.93 \mathrm{E}-05$ \\
\hline 38 & 750000 & 40.3 & 40.21286 & 0.750255 & 1513.983 & $5.93 \mathrm{E}-05$ \\
\hline 39 & 755000 & 40.42 & 40.50429 & 0.560421 & 1521.005 & $5.78 \mathrm{E}-05$ \\
\hline
\end{tabular}




\begin{tabular}{|c|c|c|c|c|c|c|}
\hline CES No. & CYCLES & A(meas.) & A(reg.) & M.C.C. & Delt. K & $\mathrm{DA} / \mathrm{DN}$ \\
\hline 40 & 760000 & 40.75 & 40.76513 & 0.63868 & 1527.297 & $5.65 \mathrm{E}-05$ \\
\hline 41 & 765000 & 41.1 & 41.06492 & 0.732167 & 1534.534 & $5.69 \mathrm{E}-05$ \\
\hline 42 & 770000 & 41.34 & 41.35038 & 0.824672 & 1541.433 & $6.08 \mathrm{E}-05$ \\
\hline 43 & 780000 & 41.99 & 41.95442 & 0.865256 & 1556.055 & $6.11 \mathrm{E}-05$ \\
\hline 44 & 790000 & 42.51 & 42.58087 & 0.888153 & 1571.258 & $6.21 \mathrm{E}-05$ \\
\hline 45 & 800000 & 43.26 & 43.21286 & 0.924115 & 1586.64 & $6.42 \mathrm{E}-05$ \\
\hline 46 & 810000 & 43.88 & 43.83239 & 0.950974 & 1601.765 & $6.61 \mathrm{E}-05$ \\
\hline 47 & 820000 & 44.49 & 44.4966 & 0.968498 & 1618.037 & $6.93 \mathrm{E}-05$ \\
\hline 48 & 840000 & 45.89 & 45.97267 & 0.976961 & 1654.427 & $7.51 \mathrm{E}-05$ \\
\hline 49 & 860000 & 47.57 & 47.60468 & 0.980865 & 1695.085 & $7.96 \mathrm{E}-05$ \\
\hline 50 & 880000 & 49.34 & 49.28429 & 0.98217 & 1737.465 & $8.06 \mathrm{E}-05$ \\
\hline 51 & 900000 & 50.98 & & & & \\
\hline 52 & 920000 & 52.52 & & & & \\
\hline 53 & 940000 & 53.98 & & & & \\
\hline
\end{tabular}




\section{Appendix D}

\section{Crack Growth Constants from Schivje}

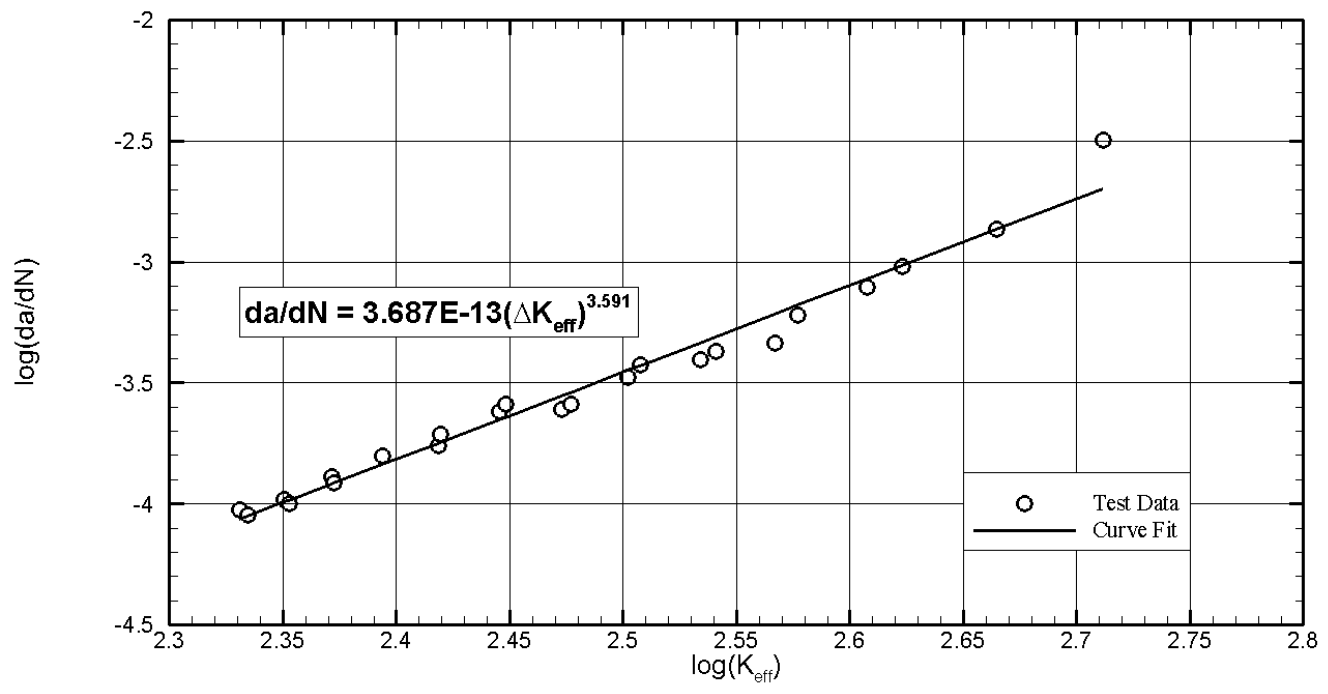

Figure D.1: Crack growth constants for Al 2024-T3 calculated using data from Schivje [17] 
Table D.1: Crack growth data from Schijve [17]

\begin{tabular}{|r|r|r|r|}
\hline$d a / d n$ & $K_{\text {eff }}$ & $\log \left(K_{\text {eff }}\right)$ & $\log (d a / d n)$ \\
\hline \hline $9.40 \mathrm{E}-05$ & 214.2939 & 2.33101 & -4.02687 \\
$1.04 \mathrm{E}-04$ & 224.148 & 2.350535 & -3.98297 \\
$1.30 \mathrm{E}-04$ & 235.1327 & 2.371313 & -3.88606 \\
$1.58 \mathrm{E}-04$ & 247.7226 & 2.393966 & -3.80134 \\
$1.94 \mathrm{E}-04$ & 262.5402 & 2.419196 & -3.7122 \\
$2.58 \mathrm{E}-04$ & 280.604 & 2.448094 & -3.58838 \\
$2.58 \mathrm{E}-04$ & 299.7664 & 2.476783 & -3.58838 \\
$3.74 \mathrm{E}-04$ & 321.6975 & 2.507448 & -3.42713 \\
$4.28 \mathrm{E}-04$ & 347.4617 & 2.540907 & -3.36856 \\
$6.02 \mathrm{E}-04$ & 377.6058 & 2.577039 & -3.2204 \\
$9.54 \mathrm{E}-04$ & 419.7944 & 2.623037 & -3.02045 \\
$3.19 \mathrm{E}-03$ & 514.6412 & 2.711505 & -2.49621 \\
$9.00 \mathrm{E}-05$ & 215.9361 & 2.334325 & -4.04576 \\
$1.00 \mathrm{E}-04$ & 225.243 & 2.352651 & -4 \\
$1.22 \mathrm{E}-04$ & 235.6461 & 2.37226 & -3.91364 \\
$1.58 \mathrm{E}-04$ & 247.7226 & 2.393966 & -3.80134 \\
$1.74 \mathrm{E}-04$ & 261.9928 & 2.418289 & -3.75945 \\
$2.42 \mathrm{E}-04$ & 278.9616 & 2.445544 & -3.61618 \\
$2.46 \mathrm{E}-04$ & 297.0631 & 2.472849 & -3.60906 \\
$3.34 \mathrm{E}-04$ & 317.8651 & 2.502243 & -3.47625 \\
$3.94 \mathrm{E}-04$ & 341.9872 & 2.53401 & -3.4045 \\
$4.64 \mathrm{E}-04$ & 368.8468 & 2.566846 & -3.33348 \\
$7.84 \mathrm{E}-04$ & 405.0127 & 2.607469 & -3.10568 \\
$1.37 \mathrm{E}-03$ & 462.0165 & 2.664657 & -2.86328 \\
\hline
\end{tabular}




\section{Appendix E}

\section{Carbon Fibre Delamination Constant}

\section{Data}

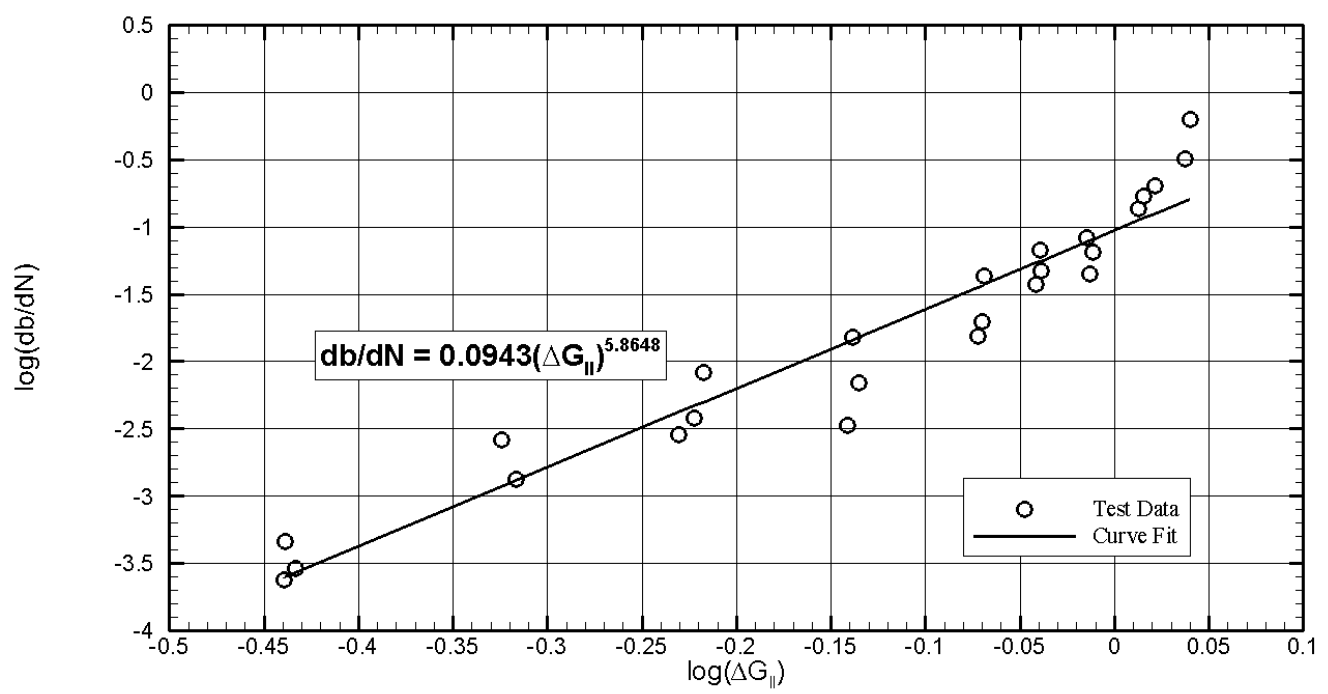

Figure E.1: Delamination growth constants for carbon fibre 
Table E.1: Delamination growth test data for carbon fibre

\begin{tabular}{|r|r|r|r|}
\hline$\Delta G_{I I}\left(M P a^{2} m m\right)$ & $d b / d N$ & $\log \left(\Delta G_{I I}\right)$ & $\log (d b / d N)$ \\
\hline 0.363396817 & 0.000239 & -0.43962 & -3.62234 \\
0.364082616 & 0.000463 & -0.4388 & -3.33461 \\
0.36851832 & 0.000292 & -0.43354 & -3.53509 \\
0.473803701 & 0.002633 & -0.3244 & -2.57953 \\
0.48252408 & 0.001325 & -0.31648 & -2.87794 \\
0.587995989 & 0.002835 & -0.23063 & -2.54741 \\
0.599384245 & 0.00378 & -0.22229 & -2.4225 \\
0.605671308 & 0.008273 & -0.21776 & -2.08235 \\
0.721710142 & 0.003347 & -0.14164 & -2.47541 \\
0.726284131 & 0.01515 & -0.13889 & -1.8196 \\
0.732685293 & 0.006961 & -0.13508 & -2.15731 \\
0.846238642 & 0.015324 & -0.07251 & -1.81463 \\
0.850415005 & 0.019954 & -0.07037 & -1.69996 \\
0.853340664 & 0.043361 & -0.06888 & -1.3629 \\
0.909013578 & 0.037584 & -0.04143 & -1.425 \\
0.913099741 & 0.066753 & -0.03948 & -1.17553 \\
0.914039214 & 0.047054 & -0.03904 & -1.32741 \\
0.966262534 & 0.082916 & -0.0149 & -1.08136 \\
0.970112557 & 0.045083 & -0.01318 & -1.34599 \\
0.973624944 & 0.064802 & -0.01161 & -1.18841 \\
1.029550899 & 0.137602 & 0.012648 & -0.86138 \\
1.036246749 & 0.169977 & 0.015463 & -0.76961 \\
1.050251467 & 0.202482 & 0.021293 & -0.69361 \\
1.089586801 & 0.31915 & 0.037262 & -0.496 \\
1.096084491 & 0.632273 & 0.039844 & -0.1991 \\
\hline
\end{tabular}

